COMMUNICATIONS IN

ANALYSIS AND GEOMETRY

Volume 8, Number 3, 575-633, 2000

\title{
Asymptotic Harmonicity of Negatively Curved Homogeneous Spaces and Their Measures at Infinity
}

\section{CHRISTOPHER CONNELL}

In this paper we explore the asymptotic geometry of negatively curved homogeneous manifolds. We characterize asymptotic harmonicity in terms of various natural measures. We also show that the Bowen-Margulis, harmonic, and Liouville measures on the unit tangent bundle and the corresponding measures on the boundary are always in the same measure class. We then show that Cheeger's constant, the Kaimanovich entropy, and the bottom of the spectrum are all maximal for these spaces. Along the way we present sharp asymptotic estimates for Jacobi fields and the Poisson and Green's kernels. Finally, we present examples showing that in general these manifolds are not asymptotically harmonic.

\section{Introduction.}

In this paper we explore the geometry of complete simply connected negatively curved homogeneous spaces, henceforth abbreviated NCHS's. The structure of non-positively curved homogeneous spaces was investigated early on by D. Alekseevskiî [1] in 1974. Much of the general structure of these spaces was described by R. Azencott and E. Wilson ([5] and [6]) and T. Wolter ([46] and [47]). They proved that these spaces all have a solvable simply transitive group of isometries of the form $A \ltimes N$, where $A$ is a (non-unique) Abelian group and $\mathrm{N}$ is the nilradical. These transitive isometry groups admit left invariant metrics which make them isometric to the manifold, although two non-isomorphic groups may be isometric. See [5] for more details.

E. Heintze [29] and J. Wolf [45] among others examined the present case of (strictly) negative curvature. Here the dimension of $A$ is one, and by homogeneity these spaces have bounded curvature $-b^{2} \leq K \leq-a^{2}<0$. E. Heintze also proved in [28] that an NCHS admits a compact quotient if and only if it is a symmetric space. In fact, the same is true if we replace compact by finite volume. 
Over the past few decades, new tools have been developed for studying the geometry and rigidity of compact manifolds of negative curvature. Both measure theory and analysis play a prominent role in these investigations. Here we draw on these ideas to develop a parallel theory for NCHS's. In particular, we define natural generalizations of the existing structures on the universal covers of compact manifolds to NCHS's. Then we attempt to apply many of the same tools for examining these structures on simply connected negatively curved manifolds with cocompact lattices (henceforth cocompact manifolds) to the case of NCHS's. However, the success of this approach rests on finding suitable replacements for compactness arguments. One especially important feature of NCHS's is that, in principle, much of the dynamics of the compact case can be replaced by the action of the transitive group of isometries. However, the compact situation does not entirely correspond with that of NCHS's. For example, some results relying on the ergodicity of the geodesic flow $g^{t}$ in the compact case do not hold for NCHS's.

Next to global symmetry one of the strongest properties a manifold can possess is being harmonic. This condition states that the mean curvature of its metric spheres is everywhere constant. The Lichnerowicz conjecture states that every harmonic manifold is a locally symmetric space. Z. Szabó in [44] verified the Lichnerowicz conjecture for compact manifolds with finite fundamental group. However, Damek and Ricci [17] found a class of harmonic non-symmetric manifolds (all of which are homogeneous spaces of so called Heisenberg type).

A manifold is asymptotically harmonic if the mean curvatures of all the horospheres are everywhere constant. Horospheres are the limits of metric spheres through a fixed point whose centers go to infinity along a geodesic ray. Hence this property is a truly asymptotic version of harmonicity. One of the two main goals of this paper is to study asymptotic harmonicity for NCHS's. Below we will give several equivalent characterizations of this property. Combining the recent results of P. Foulon and F. Labourie [22] together with two remarkable papers of Y. Benoist, P. Foulon, and F. Labourie [10] and Besson, Courtois, and Gallot [12] proves that any negatively curved compact asymptotically harmonic manifold is in fact symmetric. Both C. Yue [50] and F. Ledrappier $[37,38]$ have done much to characterize compact asymptotically harmonic manifolds in terms of their measure theoretic properties and other features of their geometry.

For any simply connected non-positively curved manifold we can define the boundary (topologically a sphere) to be equivalence classes of geodesics. Two geodesic rays are equivalent if they stay a bounded distance away from 
each other for all time. Note that we can identify measures on the boundary sphere with measures on the unit tangent sphere at any point $x \in M$ via the map which projects along geodesics originating at $x$.

We will define three important measure classes on the boundary sphere of an NCHS, in analogy with the case of cocompact manifolds. They are the Patterson-Sullivan measures $\mu_{x}$, spherical harmonic measures $\nu_{x}$, and visual measures $\lambda_{x}$ for each $x \in M$. As we will see, certain properties of these measures are closely tied in to the asymptotic geometry of the manifold. From these three measures one constructs (see section 3 ) the Bowen-Margulis measure $\mu$, harmonic measure $\nu$, and Liouville measure $\lambda$ respectively on the unit tangent bundle. These three measures are invariant under the geodesic flow and derivatives of isometries. For the weak stable, weak unstable, strong stable, and strong unstable foliations there are measures which are invariant under diffusion on the corresponding foliation. We denote the measures respectively by $\omega^{s s}, \omega^{s}, \omega^{s u}$, and $\omega^{u}$, and they are referred to (regrettably) as the harmonic measures for their respective foliation. Another natural object to consider is the flip map $F: S M \rightarrow S M$ defined by $F(v)=-v$, and for each $x \in M$ this induces a corresponding map on $\partial M$. Naturally, the pullback of a measure under the flip map is called the flip of the measure.

We present here the three main theorems of the paper for convenience. For cocompact manifolds A. Katok and D. Sullivan independently conjectured (see [9]) that if any two of the Bowen-Margulis, harmonic, or Liouville measures coincide then the manifold is a locally symmetric space. In the cocompact case these measures are all ergodic with respect to the geodesic flow, and so the conjecture is equivalent to asking that any two of the measures be in the same measure class since they would then coincide by flow-invariance. Katok proved this conjecture in dimension 2. In the non-compact case any two ways to pose this conjecture are distinct. In the first theorem we prove the analogue of the second statement of the Katok-Sullivan conjecture is false for NCHS's. We also show a somewhat surprising relationship between the Kaimanovich Entropy $\beta$, the exponential volume growth rate $\mathrm{h}$, the Cheeger constant $c_{M}$, and the bottom of the spectrum $\lambda_{1}$ (see Sections 2 and 5 for definitions).

Theorem 1. For any NCHS M, we have the following

1. For all $x \in M$ the measures $\mu_{x}, \nu_{x}, \lambda_{x}$ and their fips are all in the same measure class.

2. The measures $\mu, \nu, \lambda, \omega^{s s}, \omega^{s}, \omega^{s u}$, and $\omega^{u}$ are all in the same measure class, and 
3. $\mathrm{h}^{2}=c_{M}^{2}=\beta=4 \lambda_{1}$, moreover we can express this constant in terms of algebraic data.

In above parts (1) and (2) we also give explicit formulas for the RadonNikodym derivatives of any two of the measures. We remark item (3) above is somewhat striking because in the cocompact case any two of these quantities coincide precisely when $M$ is asymptotically harmonic [50]. A consequence of the last item of the theorem is that $M$ has no discrete spectrum. We discuss this in Section 2.

In the second theorem we show that equality of some of these measures implies asymptotic harmonicity as in the cocompact case. For what follows, $U(v)$ denotes the second fundamental form of the horosphere in the direction of $v$, and $\tau(v)$ is the derivative of the Poisson kernel along a geodesic through $v \in S M$ (see Sections 2 and 4 for details).

Theorem 2. For any NCHS M, the following are equivalent:

1. $\operatorname{tr} U$ and $\operatorname{tr} U^{2}$ are both flip invariant,

2. $\operatorname{tr} U$ is flip invariant and any one of $\mu_{x}, \omega^{s s}$, or $\omega^{s u}$ is flip invariant,

3. $\tau$ is flip invariant and any one of $\nu_{x}, \omega^{s}$, or $\omega^{u}$ is flip invariant,

4. For any $x$, any two of the measures $\mu_{x}, \nu_{x}$, or $\lambda_{x}$ coincide,

5. Any two of the measures $\lambda, \omega^{s s}$, or $\omega^{s}$ coincide,

6. Any two of the measures $\lambda, \omega^{s u}$, or $\omega^{u}$ coincide,

7. Either $\tau(v)=\mathrm{h}$ or $\tau(v)=\operatorname{tr} U(v)$, and

8. $M$ is asymptotically harmonic.

It is worth remarking that the proof of the equivalence of conditions 1 and 8 in the above theorem does not depend upon the homogeneity of $M$, and holds true for general negatively curved manifolds. Most of other conditions have been shown to be equivalent in the case of cocompact manifolds (see C. Yue [50]).

Lastly, Theorem 3 shows how equality (up to scalar) of the measures $\mu$, $\nu$, and $\lambda$ are related to various geometric implications.

Theorem 3. For any NCHS $M$, we have the following relationships

1. The following are all equivalent, 
(a) for any $x \in M$ any one of $\frac{d \mu_{x}}{d F_{*} \mu_{x}}$, $\frac{d \mu_{x}}{d \lambda_{x}}$, $\frac{d \omega^{s s}}{d \lambda}$, or $\frac{d \omega^{s u}}{d \lambda}$, is continuous,

(b) for all $v \in S M, \int_{-\infty}^{\infty} \operatorname{tr} U\left(g^{t} v\right)-\mathrm{h} d t=0$,

(c) $\lambda$ is a multiple of $\mu$.

2. The following are all equivalent,

(a) for any $x \in M$ any one of $\frac{d \nu_{x}}{d F_{*} \nu_{x}}, \frac{d \nu_{x}}{d \lambda_{x}}, \frac{d \omega^{s}}{d \lambda}$, or $\frac{d \omega^{u}}{d \lambda}$, is continuous,

(b) for all $v \in S M, \int_{-\infty}^{\infty} \operatorname{tr} U\left(g^{t} v\right)-\tau\left(g^{t} v\right) d t=0$,

(c) $\lambda$ is a multiple of $\nu$.

3. The following are all equivalent,

(a) for any $x \in M$ any one of $\frac{d \nu_{x}}{d \mu_{x}}$, $\frac{d \omega^{s}}{d \omega^{s s}}$, or $\frac{d \omega^{u}}{d \omega^{s u}}$, is continuous,

(b) for all $v \in S M, \int_{-\infty}^{\infty} \tau\left(g^{t} v\right)-\mathrm{h} d t=0$,

(c) $\mu$ is a multiple of $\nu$.

We organize the paper into seven sections, consisting of the three main results and some examples. Section 2 presents background material and proves some basic results about volume growth and asymptotic behavior. In Section 3 we establish the measure theory and describe the measures in multiple ways. In Sections 4 through Section 6 we prove Theorems 1-3. Section 4 focuses on integral formulas to establish certain relationships between the measures to obtain Theorem 1. In Section 5 we establish some of the formulas for Theorem 2 as well as find other information about these measures. Section 6 finishes up the results for the harmonic measures and compiles the three theorems. We end the paper in Section 7 with an explicit class of examples of NCHS's which are not asymptotically harmonic demonstrating that none of the criteria of Theorem 2 are trivially satisfied. A more subtle question is whether there exist asymptotically harmonic NCHS's which are not harmonic.

\section{Acknowledgments.}

I would like to thank Lizhen Ji for reading parts of this manuscript. I especially wish to thank my thesis advisor, Ralf Spatzier, for his excellent oversight and for very many insightful discussions without which this paper would not be possible. 


\section{Entropy, Volume Growth, and the Cheeger Constant.}

\subsection{Preliminaries.}

We will begin by fixing our notation. Henceforth we will always denote our NCHS by $M$ and $S M$ will be its unit tangent bundle with geodesic flow $g^{t}$.

Let $P: S M \rightarrow M$ denote the projection of $S M$ onto $M$. The distance function on $M$ and the Sasaki distance on $S M$ will both be denoted by $d(\cdot, \cdot)$ and may be distinguished by the context.

Associated to $g^{t}$ are the strong stable and strong unstable manifolds

$$
\mathcal{W}^{s s}(v)=\left\{w \in S M \mid d\left(g^{t} v, g^{t} w\right) \stackrel{t \rightarrow \infty}{\longrightarrow} 0\right\}
$$

and

$$
\mathcal{W}^{s u}(v)=\left\{w \in S M \mid d\left(g^{t} v, g^{t} w\right) \stackrel{t \rightarrow-\infty}{\longrightarrow} 0\right\} .
$$

We define the (weak) stable and (weak) unstable manifolds $\mathcal{W}^{s}(v)=$ $\cup_{t} \mathcal{W}^{s s}\left(g^{t} v\right)$ and $\mathcal{W}^{u}(v)=\cup_{t} \mathcal{W}^{s u}\left(g^{t} v\right)$ respectively.

The following description can be found in [29]. Fixing a point $o \in M$ we may identify (the orbit) $M=\mathrm{G} \cdot o$ with the simply transitive solvable group of isometries $G=A \ltimes N$ with some left invariant metric. Let $\mathfrak{g}=\mathfrak{a}+\mathfrak{n}$ be the Lie algebra of $G$. Without changing the isometry class of the corresponding left invariant metric we may choose the inner product on $\mathfrak{g}$ so that $\mathfrak{a}$ is orthogonal to $\mathfrak{n}$. The orbit $\mathrm{N} \cdot o$ of the nilradical sits in $M$ as a horosphere. Let $v_{\mathbf{a}} \in S_{o} M$ be the inward pointing tangent vector perpendicular to $\mathrm{N} \cdot o$ at the point $o$, i.e. $P \mathcal{W}^{s s}\left(v_{\mathbf{a}}\right)=\mathrm{N} \cdot o$. We will call $v_{\mathbf{a}}$ the abelian direction. We will abuse notation and also consider $v_{\mathbf{a}}$ to be the corresponding element of $\mathfrak{g}$. Then in fact $\mathfrak{a}=\mathbb{R} v_{\mathbf{a}}$, and we will denote the elements of $\mathrm{A}$ as $a^{t}=\exp t v_{\mathbf{a}}$, parametrized by $t \in \mathbb{R}$. The one parameter subgroup $a^{t} \cdot o$ and its flip $a^{-t} \cdot o$ are geodesics and the only geodesic one parameter subgroups. Furthermore, the eigenvalues of $\operatorname{ad}_{v_{\mathbf{a}}}$ have positive real parts. We refer the reader to [18] for further properties and details of the Lie group structure of $\mathrm{G}$.

For each $x \in M$ the standard spherical Lebesgue measure on $S_{x} M$ will be denoted by $\lambda_{x}$.

For a fixed left invariant metric on $M$ the connection restricted to left invariant vector fields is given by the formula

$$
\nabla_{X} Y=\frac{1}{2}\left([X, Y]-\operatorname{ad}_{X}^{*}(Y)-\operatorname{ad}_{Y}^{*}(X)\right)
$$

where $\operatorname{ad}^{*}$ denotes the adjoint of ad. Since $\mathfrak{n}$ is nilpotent and $\mathfrak{a}$ and $\mathfrak{n}$ are orthogonal, we have $\nabla_{v_{\mathbf{a}}} X=\frac{1}{2}\left(\operatorname{ad}_{v_{\mathbf{a}}}-\operatorname{ad}_{v_{\mathbf{a}}}^{*}\right)(X)$ and $\nabla_{X} v_{\mathbf{a}}=$ 
$-\frac{1}{2}\left(\operatorname{ad}_{v_{\mathbf{a}}}^{*}+\operatorname{ad}_{v_{\mathbf{a}}}\right)(X)$ as two special cases. In particular we see that A corresponds to a geodesic in $M$.

The geometric boundary of $M$ (denoted $\partial M$ ) defined earlier as equivalence classes of geodesic rays induces a compactification of $M$ where the topology on the boundary comes from geodesic cones based at points in $M$. Anderson and Schoen (see [4] or [2]) proved that for any simply connected manifold with pinched negative curvature the geometric boundary coincides with the Martin boundary, and it carries a natural Hölder structure.

For any $v \in S M, v(t) \stackrel{\text { def }}{=} P g^{t} v$ will denote the unit speed geodesic in the direction of $v$ at time $t$. The points on $\partial M$ corresponding to the equivalence classes of the geodesic rays $v(t)$ and $-v(t)$ will be denoted by $v(\infty)$ and $v(-\infty)$ respectively. For $x \neq y \in M$, we will also denote by $v_{x, y}$ the unique element of $S_{x} M$ such that the geodesic $v_{x, y}(t)$ passes through $y$ for some $t>0$. Similarly, $v_{x, \xi}$ is the unique vector such that $v_{x, \xi}(\infty)=\xi \in \partial M$. By [4] there is a natural Hölder continuous projection map $\pi_{x}:(M \backslash\{x\}) \cup$ $\partial M \rightarrow S_{x} M$ defined by $\pi_{x}(y)=v_{x, y}$. Let $\rho_{v}(y) \stackrel{\text { def }}{=} \lim _{t \rightarrow \infty} d(v(t), y)-t$ be the Busemann function associated to the point $v(\infty)=\xi$ with basepoint $v(0)=x$. We will also denote this by $\rho_{x, \xi}(y)$.

A family of linear maps $L(t): v^{\perp} \rightarrow\left(g^{t} v\right)^{\perp}$ is called Jacobi tensor along $v(t)$ if $L(t) w$ is a Jacobi field for each $w \in v^{\perp}$. A Jacobi field $J(t)$ is called stable (resp. unstable) if $J(\infty)=0$ (resp. $J(-\infty)=0$ ). There are Jacobi tensors $\Lambda(v, t), E(v, t): v^{\perp} \rightarrow\left(g^{t} v\right)^{\perp}$ such that for $w \in v^{\perp}, E(v, t) w$ (resp. $\Lambda(v, t) w$ ) is the stable (resp. unstable) Jacobi field $J(t)$ with $J(0)=w$. We call $E(v, t)$ (resp. $\Lambda(v, t)$ ) the stable (resp. unstable) Jacobi tensors along the geodesic $v(t)$.

For $v \in S M$ the stable, $E^{s}(v)$, and unstable, $E^{u}(v)$, distributions on $S M$ with respect to $g^{t}$ are the tangent spaces to the $\mathcal{W}^{s s}(v)$ and $\mathcal{W}^{s u}(v)$. After identifying $E^{s}(v)$ (resp. $E^{u}(v)$ ) with $T_{P v} P \mathcal{W}^{s s}(v)=v^{\perp}$ (resp. $T_{P v} P \mathcal{W}^{s u}(v)=v^{\perp}$ ), the corresponding stable and unstable Jacobi tensors along a geodesic $v(t)$ are given by

$$
\left.E(v, t) \stackrel{\text { def }}{=} d g^{t}\right|_{E^{s}(v)} \text { and }\left.\Lambda(v, t) \stackrel{\text { def }}{=} d g^{t}\right|_{E^{u}(v)}
$$

respectively. Applying the chain rule gives us the relations

$$
\Lambda(v, t+s)=\Lambda\left(g^{s} v, t\right) \Lambda(v, s) \text { and } E(v, t+s)=E\left(g^{s} v, t\right) E(v, s) .
$$

Since these tensors both satisfy the Jacobi equation, it is easy to see that $E(v, t)=\Lambda(-v,-t)$ once we identify $(-v)^{\perp}$ with $v^{\perp}$. The (symmetric, positive definite) second fundamental form of the horosphere $P \mathcal{W}^{s s}(v)$ at 
the point $P v \in M$ is denoted by $U(v)$. It is related to the stable and unstable Jacobi tensors by

$$
\begin{aligned}
U\left(g^{t} v\right) & =-E(v, t)^{\prime} E^{-1}(v, t) \quad \text { and } \\
U\left(-g^{t} v\right) & =\Lambda(v, t)^{\prime} \Lambda^{-1}(v, t) .
\end{aligned}
$$

From Proposition 2.8 of [14] (for example) it follows that

$$
\begin{aligned}
& \operatorname{det} E(v, t)=e^{\int_{0}^{t}-\operatorname{tr} U\left(g^{s} v\right) d s} \quad \text { and, } \\
& \operatorname{det} \Lambda(v, t)=e^{\int_{0}^{t} \operatorname{tr} U\left(-g^{s} v\right) d s} .
\end{aligned}
$$

In the literature $U(v)$ is more commonly defined to be the second fundamental form of $P \mathcal{W}^{s u}(v)$, i.e. our $U(-v)$. However for the sake of consistency with some of the propositions we have adopted the viewpoint of $\mathrm{C}$. Yue in [50].

Define the volume element in polar coordinates to be $\mathrm{V}(x, y)=$ $t^{n-1} \operatorname{det} d\left(\exp _{x}\right)_{\exp _{x}^{-1}(y)}$ where $t=d(x, y)$. For $v \in S M$, define $A(v, t)$ to be the Jacobi (Lagrange) tensor such that $A(v, 0)=0$ and $A^{\prime}(v, 0)=I d$. Observe that $A(v, t)=\left.t \cdot d\left(\exp _{P v}\right)_{v t}\right|_{v^{\perp}}$. Then the volume element $\mathrm{V}(v, t)=$ $\mathrm{V}(v(0), v(t))$ along a geodesic $v(t)$ can be described as $\mathrm{V}(v, t)=\operatorname{det} A(v, t)$. In terms of the Jacobi tensors $\Lambda$ and $E$, the tensor $A$ can be uniquely described as

$$
A(v, t)=(\Lambda(v, t)-E(v, t))\left(\Lambda^{\prime}(v, 0)-E^{\prime}(v, 0)\right)^{-1} .
$$

This follows from observing that $A(v, t)$ formally satisfies the given properties and the Jacobi equation. It is well defined since $\Lambda^{\prime}(v, 0)$ is positive definite and $E^{\prime}(v, 0)$ is negative definite. Furthermore, it is analytic on $S M \times \mathbb{R}$ since the map $\exp _{P v}(w t)$ is analytic in $v, w$, and $t$.

Remark 2.1. By examining the above formulas notice that $A(-v,-t)=$ $-A(v, t)$ and $\Lambda^{\prime}(v, 0)-E^{\prime}(v, 0)=U(-v)+U(v)$. Hence we may write

$$
\mathrm{V}(v, t)=\frac{\operatorname{det}(\Lambda(v, t)-E(v, t))}{\operatorname{det}(U(v)+U(-v))} .
$$

Also since the eigenvalues of $\Lambda$ are exponentially increasing and those of $E(v, t)$ are exponentially decreasing, we have

$$
\lim _{t \rightarrow \infty} \frac{\mathrm{V}(v, t)}{\operatorname{det} \Lambda(v, t)}=\frac{1}{\operatorname{det}(U(v)+U(-v))} .
$$


For what follows, denote by $B(x, r)$ the metric ball of radius $r$ centered at $x$.

Definition 2.2. The volume growth entropy $\mathrm{h}$ for $M$ is the exponential growth rate of the volume of balls:

$$
\mathrm{h} \stackrel{\text { def }}{=} \limsup _{r \rightarrow \infty} \frac{\log \operatorname{Vol} B(x, r)}{r} .
$$

Note that $\mathrm{h}$ is independent of $x$ by the triangle inequality.

\subsection{Asymptotic Control of Jacobi Fields and Volume Growth.}

The purpose of this subsection is to prove the following

Theorem 2.3. There are constants $C_{5}, C_{6} \geq 1$ independent of $v \in S M$ such that for all $T>0$,

$$
\frac{1}{C_{5}} \leq \operatorname{det} \Lambda(v, T) e^{-\operatorname{tr}\left(\operatorname{ad}_{v_{\mathbf{a}}}\right) T} \leq C_{5},
$$

and

$$
\frac{1}{C_{6}} \leq \mathrm{V}(v, T) e^{-\operatorname{tr}\left(\operatorname{ad}_{v_{\mathbf{a}}}\right) T} \leq C_{6}
$$

Furthermore, as $T \rightarrow \infty$ (resp. $T \rightarrow-\infty), \operatorname{det} \Lambda(v, T) e^{-\operatorname{tr}\left(\operatorname{ad}_{\mathbf{a}}\right) T}$ and $\mathrm{V}(v, T) e^{-\operatorname{tr}\left(\operatorname{ad}_{v_{\mathbf{a}}}\right) T}$ converge exponentially fast to continuous functions on $S M \backslash \mathcal{W}^{s}\left(v_{\mathbf{a}}\right)$ (resp. $\left.S M \backslash \mathcal{W}^{u}\left(-v_{\mathbf{a}}\right)\right)$.

Remark 2.4. See Section 7 for examples of spaces where

$$
\lim _{t \rightarrow \infty} \operatorname{det} \Lambda(v, T) e^{-\operatorname{tr}\left(\operatorname{ad}_{v_{\mathbf{a}}}\right) T}
$$

and

$$
\lim _{t \rightarrow \infty} \mathrm{V}(v, T) e^{-\operatorname{tr}\left(\operatorname{ad}_{v_{\mathbf{a}}}\right) T}
$$

are not continuous at $\mathcal{W}^{s}\left(v_{\mathbf{a}}\right)$.

Before proving this theorem, we will present two simple corollaries.

Corollary 2.5. The exponential volume growth rate satisfies $\mathrm{h}=\operatorname{tr} \operatorname{ad}_{v_{\mathbf{a}}}$. 
Proof. Define the equivalence relation $\simeq$ by declaring $A \simeq B$ if there exists a constant $C>1$ such that $\frac{1}{C} B<A<C B$. Then using the bounds in Theorem 2.3 we obtain the following estimate for $\operatorname{Vol}\left(B_{r}(x)\right)$.

$$
\begin{aligned}
\operatorname{Vol}\left(B_{r}(x)\right) & =\int_{S_{x} M} \int_{0}^{r} \mathrm{~V}(v, t) \mathrm{dtd} \lambda_{\mathrm{x}} \\
& \simeq \int_{S_{x} M} \int_{0}^{r} e^{\operatorname{trad}_{v_{\mathbf{a}}} t} \mathrm{dtd} \lambda_{\mathbf{x}} \\
& \simeq e^{\operatorname{trad}_{v_{\mathbf{a}}} r} .
\end{aligned}
$$

The statement of the corollary follows immediately.

Definition 2.6. For negatively curved manifolds, the Margulis function is defined to be

$$
C_{\mathrm{Mar}}(x) \stackrel{\text { def }}{=} \limsup _{r \rightarrow \infty} \frac{\operatorname{Vol}\left(S_{r}(x)\right)}{e^{\mathrm{h} r}} .
$$

For compact manifolds the Margulis function is always finite and the limsup can be replaced by lim. However, A. Katok showed that in dimension 2 it is constant only for symmetric surfaces, and the corresponding conjecture remains open in higher dimensions [50]. In contrast, for NCHS's we always have

Corollary 2.7. The limit $\lim _{r \rightarrow \infty} \frac{\mathrm{Vol}\left(S_{r}(x)\right)}{e^{\mathrm{h} r}}$ exists and is a positive constant $C_{\text {Mar }}=C_{\text {Mar }}(x)$.

Proof. Writing $\operatorname{Vol}\left(S_{r}(x)\right)$ in polar coordinates for any $r>0$ yields

$$
\frac{\mathrm{Vol}\left(S_{r}(x)\right)}{e^{\operatorname{tr}\left(\operatorname{ad}_{v_{\mathbf{a}}}\right) r}}=\int_{S_{x} M} \mathrm{~V}(v, r) e^{-\operatorname{tr}\left(\operatorname{ad}_{v_{\mathbf{a}}}\right) r} \mathrm{~d} \lambda_{\mathbf{x}}
$$

By Theorem 2.3, for any $v \in S_{x} M$ the integrand in the expression above converges exponentially quickly to a positive value, bounded independent of $v$. By the Lebesgue Dominated Convergence Theorem the above integral converges as $r \rightarrow \infty$ to a finite value which by definition must be $C_{\mathrm{Mar}}(x)$. Now observe that $\operatorname{Vol}\left(S_{r}(x)\right)=\operatorname{Vol}\left(S_{r}(o)\right)$ since there is an isometry which carries $S_{r}(o)$ onto $S_{r}(x)$. Hence $C_{\mathrm{Mar}}(x)$ is constant.

In order to prove the theorem we must first control Jacobi Fields along the abelian geodesic. Set $\operatorname{ad}_{v_{\mathbf{a}}}=D+S$ where $D$ and $S$ are symmetric and (resp.) skew-symmetric endomorphisms of $\mathfrak{g}$ (i.e acting on left invariant 
fields). For any $v \in S M$ define the curvature operator to be the endomorphism of $T_{P v} M$ given by $R(v)=R(v, \cdot) v$. Also denote by $\|_{t}$ parallel transport along the geodesic $v_{\mathbf{a}}(t)$.

Lemma 2.8. In the abelian direction, the stable Jacobi tensor is

$$
E\left(v_{\mathbf{a}}, t\right)=\|_{t} e^{S t} e^{-a d_{v_{\mathbf{a}}} t} .
$$

The unstable Jacobi tensor is given by

$$
\Lambda\left(v_{\mathbf{a}}, t\right)=\|_{t} e^{S t} e^{B t}
$$

where $B$ is the unique solution of the linear equation $(B+2 S) B=(D-$ $S)(D+S)$ with the same eigenvalues as $\operatorname{ad}_{v_{\mathbf{a}}}$ (If $\operatorname{ad}_{v_{\mathbf{a}}}$ is a normal operator then $B=a d_{v_{\mathbf{a}}}^{*}$ ). Furthermore, the second fundamental form at $g^{t} v_{\mathbf{a}}$ of the horosphere corresponding to $\mathcal{W}^{s s}\left(g^{t} v_{\mathbf{a}}\right)$ is $U\left(g^{t} v_{\mathbf{a}}\right)=\left\|_{t} e^{S t} D e^{-S t}\right\|_{-t}$, and similarly $U\left(-g^{t} v_{\mathbf{a}}\right)=\left\|_{t} e^{S t}(B+S) e^{-S t}\right\|_{-t}$.

Proof. For any left invariant field $X$ along $v_{\mathbf{a}}(t)$ we have that $\nabla_{v_{\mathbf{a}}} X=S X$ (from 2.1). Hence $X$ restricted to the geodesic $v_{\mathbf{a}}(t)$ is given by $X\left(v_{\mathbf{a}}(t)\right)=\|_{t}$ $e^{S t} X(o)$. Therefore the curvature operator at $g^{t} v_{\mathbf{a}}$ is $R\left(g^{t} v_{\mathbf{a}}\right)=-\|_{t}$ $e^{S t}\left(D^{2}+[D, S]\right) e^{-S t} \|_{-t}$, since by 1.4 of $[18]$ we have $R\left(v_{\mathbf{a}}\right)=-\left(D^{2}+[D, S]\right)$. Here again we are identifying $\mathfrak{g}$ with $S_{o} M$.

For any Jacobi field $J_{v_{\mathbf{a}}}(t)$ along $v_{\mathbf{a}}(t)$ the Jacobi equation then becomes

$$
\nabla_{v_{\mathbf{a}}} \nabla_{v_{\mathbf{a}}} J_{v_{\mathbf{a}}}(t)-\left\|_{t} e^{S t}\left(D^{2}+[D, S]\right) e^{-S t}\right\|_{-t} J_{v_{\mathbf{a}}}(t)=0 .
$$

We now search for solutions to this equation of the form $J_{v_{\mathbf{a}}}(t)=\|_{t}$ $e^{S t} L(t) J_{v_{\mathbf{a}}}(0)$ for some parametrized endomorphisms $L(t)$ of $v_{\mathbf{a}}{ }^{\perp}$. Explicitly,

$$
\nabla_{v_{\mathbf{a}}} J_{v_{\mathbf{a}}}(t)=\left\|_{t} \frac{d}{d t} e^{S t} L(t) J_{v_{\mathbf{a}}}(0)=\right\|_{t} e^{S t}\left(S L(t)+L^{\prime}(t)\right) J_{v_{\mathbf{a}}}(0) .
$$

So the Jacobi equation above becomes

$$
0=\left\|_{t} e^{S t}\left(S^{2} L+2 S L^{\prime}+L^{\prime \prime}\right) J_{v_{\mathbf{a}}}(0)-\right\|_{t} e^{S t}\left(D^{2}+D S-S D\right) L J_{v_{\mathbf{a}}}(0) .
$$

The second order constant coefficient equation $L^{\prime \prime}+2 S L^{\prime}-\left(D^{2}+D S-S D-\right.$ $\left.S^{2}\right) L=0$ has solutions which correspond to the various Jacobi fields. For the stable and unstable fields, the cocycle properties imply that $L(t+s)=$ $L(t) \cdot L(s)$. Hence, $L(t)=e^{B t}$ for some matrix $B$.

After substituting $L=e^{B t}$, we are reduced to solving the equation $B^{2}+$ $2 S B+S^{2}=D^{2}+D S-S D$ or $(B+2 S) B=(D-S)(D+S)$ which has one 
solution $B=-D-S=-\operatorname{ad}_{v_{\mathbf{a}}}$. This solution gives all stable Jacobi fields, $J_{v_{\mathbf{a}}}(t)=E\left(v_{\mathbf{a}}, t\right) J_{v_{\mathbf{a}}}(0)$. The reason being that the eigenvalues of $a d_{v_{\mathbf{a}}}$ have negative real parts (see Heintze [29]) which implies that $e^{-\operatorname{ad}_{v_{\mathbf{a}}} t} \stackrel{t \rightarrow \infty}{\longrightarrow} 0$. Similarly, the linear operator $B$ corresponding to the unstable Jacobi fields $J_{v_{\mathbf{a}}}(t)=\Lambda\left(v_{\mathbf{a}}, t\right) J_{v_{\mathbf{a}}}(0)$ will be a solution of $(B+2 S) B=(D-S)(D+S)$ with all eigenvalues having positive real parts. The existence and uniqueness of this solution are a consequence of the existence and uniqueness of $\Lambda\left(v_{\mathbf{a}}, t\right)$.

To examine this solution substitute $B$ with $B=C-\operatorname{ad}_{v_{\mathbf{a}}}$ then the equation becomes

$$
C B-\operatorname{ad}_{v_{\mathrm{a}}}^{*} C=0 .
$$

Suppose first that $C$ is not invertible. By formula 2.5, $B$ leaves $\operatorname{ker}(C)$ invariant, but then $-\left.\operatorname{ad}_{v_{a}}\right|_{\operatorname{ker}(C)}=\left.(B-C)\right|_{\operatorname{ker}(C)}=\left.B\right|_{\operatorname{ker}(C)}$. This is impossible since the eigenvalues of $-\operatorname{ad}_{v_{\mathbf{a}}}$ and $B$ have negative and positive real parts respectively. Hence $C$ is invertible and we may write equation 2.5 as $B=C^{-1} \operatorname{ad}_{v_{\mathbf{a}}}^{*} C$ which shows that the eigenvalues of $B$ are identical to those of $\operatorname{ad}_{v_{\mathbf{a}}}$. Unfortunately, there is no simple expression for this solution in terms of $D$ and $S$, unless $S$ commutes with $D$.

Lastly, using the relation $U\left(-g^{t} v\right)=\Lambda^{\prime}(v, t) \Lambda^{-1}(v, t)$ from 2.2 and a simple computation results in the given formula for $U\left( \pm g^{t} v_{\mathbf{a}}\right)$.

Corollary 2.9. We have $\operatorname{det} \Lambda\left( \pm v_{\mathbf{a}}, t\right)=e^{\operatorname{trad}_{v_{\mathbf{a}}} t}$ and $\operatorname{det} E\left( \pm v_{\mathbf{a}}, t\right)=$ $e^{-\operatorname{trad}_{v_{\mathbf{a}}} t}$

Proof. From the above lemma, we have the following expression for the stable and unstable Jacobi fields $E\left(v_{\mathbf{a}}, t\right)=\|_{t} e^{S t} e^{-\operatorname{ad}_{v_{\mathbf{a}}} t}$ and $\Lambda\left(v_{\mathbf{a}}, t\right)=\|_{t} e^{S t} e^{B t}$. Recall that the eigenvalues of $B$ are those of $\operatorname{ad}_{v_{\mathbf{a}}}$ and that $e^{S t}$ and $\|_{t}$ act orthogonally. Taking determinants, we obtain $\operatorname{det} \Lambda\left(v_{\mathbf{a}}, t\right)=\operatorname{det} \|_{t} e^{S t} e^{B t}=$ $e^{\operatorname{tr} B t}=e^{\operatorname{trad} v_{\mathbf{a}} t}$ and similarly $\operatorname{det} E\left(v_{\mathbf{a}}, t\right)=e^{-\operatorname{trad}_{v_{\mathbf{a}}} t}$. Lastly we use the relationships $\Lambda\left( \pm v_{\mathbf{a}}, t\right)=E\left(\mp v_{\mathbf{a}},-t\right)$ to establish the other two cases.

Now recall that $-b^{2} \leq K \leq-a^{2}<0$. Let $d$ denote the Sasaki metric or the metric on $M$ depending on the context. In the rest of the section, define $w_{v}^{+} \stackrel{\text { def }}{=} \mathcal{W}^{s s}(v) \cap \mathcal{W}^{u}\left(-v_{\mathbf{a}}\right)$ for $v \in S M \backslash \mathcal{W}^{s}\left(v_{\mathbf{a}}\right)$ and $w_{v}^{-} \stackrel{\text { def }}{=} \mathcal{W}^{s u}(v) \cap \mathcal{W}^{s}\left(v_{\mathbf{a}}\right)$ for $v \in S M \backslash \mathcal{W}^{u}\left(-v_{\mathbf{a}}\right)$. The $w_{v}^{ \pm}$are well defined since the corresponding stable and unstable leaves intersect in one point. We then can make the following estimates 
Lemma 2.10. Let $f: S M \rightarrow \mathbb{R}$ be any $\alpha$-Hölder continuous function. Then there are constants $C_{1}, C_{2}>0$ such that for any $v \in S M$ and all $t \geq 0$, either $d\left(v, w_{v}^{+}\right) \leq C_{1}$ and $\left|f\left(g^{t} v\right)-f\left(g^{t} w_{v}^{+}\right)\right| \leq C_{2} e^{-\alpha a t}$ or $d\left(v, w_{v}^{-}\right) \leq C_{1}$ and $\left|f\left(g^{-t} v\right)-f\left(g^{-t} w_{v}^{-}\right)\right| \leq C_{2} e^{-\alpha a t}$.

Proof. We first claim that there is a constant $C_{1}>0$ such that for all $v \in S M$ the function $\min \left\{d\left(v, w_{v}^{+}\right), d\left(v, w_{v}^{-}\right)\right\}$is less than $C_{1}$. By homogeneity it is enough to prove the lemma for $v \in S_{o} M$, since we can translate the vector $v$ to $S_{o} M$ by an isometry $g \in \mathrm{G}$ which leaves $\mathcal{W}^{u}\left(-v_{\mathbf{a}}\right)$ and $\mathcal{W}^{s}\left(v_{\mathbf{a}}\right)$ invariant and hence $d g w_{v}^{ \pm}=w_{d g v}^{ \pm}$. Recall from the definition of $w_{v}^{ \pm}$ that the functions $d\left(v, w_{v}^{+}\right)$and $d\left(v, w_{v}^{-}\right)$are continuous on $S_{o} M \backslash\left\{v_{\mathbf{a}}\right\}$ and $S_{o} M \backslash\left\{-v_{\mathbf{a}}\right\}$ respectively. The function $\min \left\{d\left(v, w_{v}^{+}\right), d\left(v, w_{v}^{-}\right)\right\}$can be extended to a continuous function on all of $S_{o} M$ since $d\left(v, w_{v}^{-}\right) \leq d\left(v, w_{v}^{+}\right)$in some neighborhood of $v_{\mathrm{a}}$ and $d\left(v, w_{v}^{+}\right) \leq d\left(v, w_{v}^{-}\right)$in some neighborhood of $-v_{\mathbf{a}}$. Hence, by compactness of $S_{o} M$ the function $\min \left\{d\left(v, w_{v}^{+}\right), d\left(v, w_{v}^{-}\right)\right\}$ achieves a maximum. Let this maximum value be the constant $C_{1}$.

Now consider the above construction in hyperbolic space of constant curvature $K=-a^{2}$. In this case there is a constant $C$ depending only on $C_{1}$ and $a$ such that for all $t \geq 0$ either $d\left(g^{t} v, g^{t} w_{v}^{+}\right) \leq C e^{-a t}$ if $d\left(v, w_{v}^{+}\right) \leq C_{1}$ or $d\left(g^{-t} v, g^{-t} w_{v}^{-}\right) \leq C e^{-a t}$ if $d\left(v, w_{v}^{-}\right) \leq C_{1}$. By the standard Toponogov comparison theorem for triangles, these same estimates hold in our case. Then by the Hölder condition there is a constant $C^{\prime}$ such that either $\left|f\left(g^{t} v\right)-f\left(g^{t} w_{v}^{+}\right)\right| \leq C^{\prime} d\left(g^{t} v, g^{t} w_{v}^{+}\right)^{\alpha} \leq C_{2} e^{-\alpha a t}$ or $\left|f\left(g^{-t} v\right)-f\left(g^{-t} w_{v}^{-}\right)\right| \leq C^{\prime} d\left(g^{t} v, g^{t} w_{v}^{-}\right)^{\alpha} \leq C_{2} e^{-\alpha a t}$ where $C_{2}=C^{\prime} C^{\alpha}$.

For any Hölder function $f: S M \rightarrow \mathbb{R}$, define

$$
I_{f}(v, T) \stackrel{\text { def }}{=}\left\{\begin{array}{cccc}
\int_{0}^{T}\left|f\left(g^{t} v\right)-f\left(g^{t} w_{v}^{+}\right)\right| d t & \text { for } & v \in S M \backslash \mathcal{W}^{s}\left(v_{\mathbf{a}}\right) \\
0 & \text { for } & v \in \mathcal{W}^{s}\left(v_{\mathbf{a}}\right) .
\end{array}\right.
$$

If we denote by $\pm \mathcal{W}^{s}\left(v_{\mathbf{a}}\right)$ the set $\mathcal{W}^{u}\left(-v_{\mathbf{a}}\right) \cup \mathcal{W}^{s}\left(v_{\mathbf{a}}\right)$, then we have the following estimates.

Corollary 2.11. If $f$ is an $\alpha$-Hölder function which is constant on the set $\pm \mathcal{W}^{s}\left(v_{\mathbf{a}}\right)$ then there is a constant $C_{3}$ such that the estimate $I_{f}(v, T) \leq C_{3}$ holds for all $v \in S M$ and all $T \geq 0$. Furthermore $\lim _{T \rightarrow \infty} I_{f}(v, T)$ always exists and is continuous for $v \in S M \backslash \mathcal{W}^{s}\left(v_{\mathbf{a}}\right)$ with $I_{f}(v, \infty)-I_{f}(v, T) \leq$ $C_{4} e^{-\alpha a T}$ for some constant $C_{4}$ which depends continuously on $v \in S M \backslash$ $\mathcal{W}^{s}\left(v_{\mathbf{a}}\right)$. Lastly, the convergence is uniformly exponentially fast on compact subsets of $S M \backslash \mathcal{W}^{s}\left(v_{\mathbf{a}}\right)$ 
Proof. Let $T_{0} \geq 0$ be the first value of $t$ such that $d\left(g^{t} v, g^{t} w_{v}^{+}\right) \leq C_{1}$ where $C_{1}$ is the constant from Lemma 2.10. Then we can split the integral up as

$$
I_{f}(v, T)=\int_{0}^{T_{0}}\left|f\left(g^{T_{0}-t} v\right)-f\left(g^{t} w_{v}^{+}\right)\right| d t+\int_{T_{0}}^{T}\left|f\left(g^{t} v\right)-f\left(g^{t} w_{v}^{+}\right)\right| d t .
$$

Since $I_{f}(v, T)$ is nondecreasing in $T$ we may assume without loss of generality that $T \geq T_{0}$. By assumption we have the equality $f\left(g^{t}\left( \pm v_{\mathbf{a}}\right)\right)=f\left(v_{\mathbf{a}}\right)$. Also, $d\left(g^{t} v, g^{t} w_{v}^{-}\right) \leq C_{1}$ for $0 \leq t \leq T_{0}$, so we have the following estimate

$$
\int_{0}^{T_{0}}\left|f\left(g^{-t}\left(g^{T_{0}} v\right)\right)-f\left(v_{\mathbf{a}}\right)\right| d t \leq \int_{0}^{T_{0}} C_{2} e^{-\alpha a t} d t .
$$

For the second integral we have the similar estimate

$$
\begin{aligned}
\int_{T_{0}}^{T}\left|f\left(g^{t} v\right)-f\left(v_{\mathbf{a}}\right)\right| d t & =\int_{0}^{T-T_{0}}\left|f\left(g^{t}\left(g^{T_{0}} v\right)\right)-f\left(v_{\mathbf{a}}\right)\right| d t \\
& \leq \int_{0}^{T-T_{0}} C_{2} e^{-\alpha a t} d t
\end{aligned}
$$

Hence

$$
I_{f}(v, T) \leq \frac{C_{2}}{\alpha a}\left(1-e^{-\alpha a T_{0}}\right)+\frac{C_{2}}{\alpha a}\left(1-e^{-\alpha a\left(T-T_{0}\right)}\right) \leq \frac{2}{\alpha a} C_{2}=C_{3} .
$$

From the above estimates we can compute for all $R \geq T$ we have

$$
\begin{aligned}
I_{f}(v, R)-I_{f}(v, T) & \leq \frac{C_{2}}{\alpha a}\left(e^{-\alpha a\left(T-T_{0}\right)}-e^{-\alpha a\left(R-T_{0}\right)}\right) \\
& =\frac{C_{2}}{\alpha a} e^{-\alpha a\left(T-T_{0}\right)}\left(1-e^{-\alpha a(R-T)}\right) .
\end{aligned}
$$

Choosing $C_{4}=\frac{C_{2}}{\alpha a} e^{\alpha a T_{0}}$, as $R \rightarrow \infty$ the second estimate follows.

By the continuous dependence of $w_{v}^{+}$on $v \in S M \backslash \mathcal{W}^{s}\left(v_{\mathbf{a}}\right)$ it follows that $T_{0}$ and hence $C_{4}$ depend continuously on $v \in S M \backslash \mathcal{W}^{s}\left(v_{\mathbf{a}}\right)$. Now observe that $I_{f}(v, T)$ is continuous in $v$ for any fixed $T$. By the continuity of $C_{4}$ the tail $I_{f}(w, \infty)-I_{f}(w, T)$ decays exponentially quickly in $T$ and uniformly in $w$ for $w$ sufficiently near $v \notin \mathcal{W}^{s}\left(v_{\mathbf{a}}\right)$. Hence, $I_{f}(v, \infty)$ is continuous for $v \in S M \backslash \mathcal{W}^{s}\left(v_{\mathbf{a}}\right)$. In the same way the uniform convergence follows from the continuity of $C_{4}$.

Before we begin the proof of the theorem we need to establish the above results for the case of $\operatorname{tr} U$. 
Lemma 2.12. The function $\operatorname{tr} U(v)$ is bounded and uniformly Hölder continuous on $S M$. Furthermore, it is constant on the set $\pm \mathcal{W}^{s}\left(v_{\mathbf{a}}\right)$.

Proof. Eberlein (see [30]) showed that the $W^{i}$ foliations are continuous for general Hadamard manifolds. Now we examine the proof of Proposition 4.4 in the appendix of [8]. The upper bound on the curvature of $M$ gives us an Anosov splitting of TSM for the geodesic flow $g^{t}$ with respect to the Sasaki metric. Since we have a transitive group of isometries on $M$ the derivatives of the flow $g^{t}$ only depend on the values at the tangent space at one base point in $M$. It follows that the derivatives of the flow are bounded. This suffices for the proof of Proposition 4.4 which then shows that the distributions $E^{i}$, for $i=s, s s, u, s u$, generating the foliations $\mathcal{W}^{i}$ are Hölder continuous.

The bundle $T S M$ splits as $T S M=T^{h} \oplus T^{v} \oplus T^{o}$ where $T^{o}$ is the one dimensional span of the geodesic spray, $T^{v}$ is the vertical bundle (i.e. the tangent bundle to the unit tangent sphere foliation), and $T^{h}$ is the horizontal bundle (orthogonal complement of $T^{v} \oplus T^{o}$ with respect to the Sasaki metric). $T_{v}^{h}$ is isomorphic to $v^{\perp} \in T_{P v} M$ via the map $d P$ and $T_{v}^{v}=T_{v} S_{P v} M$ is also canonically isomorphic (and isometric) to $v^{\perp}$ via the connection map. We define $\mathcal{L}: T^{h} \rightarrow v^{\perp} \rightarrow T^{v}$ to be the induced smooth bundle isomorphism. Since $v^{\perp}=T_{P v} P \mathcal{W}^{s s}(v)$, the second fundamental form $U(v)$ of $P \mathcal{W}^{s s}(v)$ can be viewed as an automorphism of $T_{v}^{v}$ (or of $T_{v}^{h}$ ). Then we can express (see Section 1 of [26]) the stable and unstable distributions in terms of $U(v)$ as,

$$
E_{v}^{s u}=\left\{X+U(-v) \mathcal{L}(X) \mid X \in T_{v}^{h}\right\} \quad \text { and } \quad E_{v}^{s s}=\left\{X-U(v) \mathcal{L} X \mid X \in T_{v}^{h}\right\}
$$

Since $\mathcal{L}$ is smooth, the regularity of $U(v)$ as a section of $\left(T^{v}\right)^{*} \otimes T^{v}$ is the same as that of $E^{s s}$ and $E^{s u}$ (see the beginning of the proof of Lemma 2.4 in [26]). In particular $\operatorname{tr} U$ is Hölder continuous as a function.

Lastly, since $\operatorname{tr} U$ is G-invariant it only depends on its values at $S_{o} M$ and hence it is bounded and uniformly Hölder. The last statement follows from $\pm \mathcal{W}^{s}\left(v_{\mathbf{a}}\right)$ being an orbit of $\mathrm{G}$.

Remark 2.13. Since the curvature tensor is analytic and G-invariant, its values only depend linearly on the degree 2 exterior algebra of the tangent space at one point. In particular, its derivatives are all uniformly bounded. Remark 3.3 of Chapter IV of [8] states that the Busemann function is $C^{\infty}$, and hence the leaves of the $\mathcal{W}^{i}$ foliation are individually $C^{\infty}$ for $i=s, s s, u, s u$. 
Proof of Theorem 2.3. Since the isometries in $\mathbf{N}$ are transitive on $\mathcal{W}^{s}\left(v_{\mathbf{a}}\right)$, $\operatorname{tr} U(v)$ is constant on $\mathcal{W}^{s}\left(v_{\mathbf{a}}\right)$ and $-\mathcal{W}^{s}\left(v_{\mathbf{a}}\right)$ separately. Furthermore $\operatorname{tr} U\left(v_{\mathbf{a}}\right)=\operatorname{tr} U\left(-v_{\mathbf{a}}\right)$ (see Lemma 2.9) so $\operatorname{tr} U$ is constant on $\pm \mathcal{W}^{s}\left(v_{\mathbf{a}}\right)$. Now in [24], using only curvature bounds, $\operatorname{tr} U$ is shown to be an $\frac{a}{b}$-Hölder function. Hence, applying Corollary 2.11 to $\operatorname{tr} U(v)$ we obtain a constant $C>0$ such that $\int_{0}^{T}\left|\operatorname{tr} U\left(g^{t} v\right)-\operatorname{tr}\left(\operatorname{ad}_{v_{\mathbf{a}}}\right)\right| d t=\int_{0}^{T}\left|\operatorname{tr} U\left(g^{t} v\right)-\operatorname{tr} U\left(v_{\mathbf{a}}\right)\right| d t \leq C$.

By equations 2.3 it follows that,

$$
\operatorname{det} \Lambda(v, T) e^{-\operatorname{tr}\left(\operatorname{ad}_{v_{\mathbf{a}}}\right) T}=e^{\int_{0}^{T}\left(\operatorname{tr} U\left(-g^{s} v\right)-\operatorname{tr}\left(\operatorname{ad}_{v_{\mathbf{a}}}\right)\right) d s} .
$$

By our estimate above for the exponent we see that this expression is bounded above and below by the uniform constants $\frac{1}{C_{5}}, C_{5}$ respectively, where $C_{5}=e^{C}$. The second bound follows from the first by Remark 2.1 .

The existence and continuity of the positive limits for these functions as $T \rightarrow \infty$ is a direct consequence of the existence and continuity of $I_{\operatorname{tr} U}(v, \infty)$ given in Corollary 2.11. The continuity of the negative limits follows in the same way since $I_{\operatorname{tr} U(v)}(v,-T)=I_{\operatorname{tr} U(-v)}(-v, T)$.

\subsection{Cheeger's Constant and the Bottom of the Spectrum.}

Let

$$
\lambda_{1}(N)=\inf \left\{\frac{\int_{N}|\nabla \phi|^{2} d m}{\int_{N} \phi^{2} d m}: \phi \in C_{0}^{\infty}(N), \phi \not \equiv 0\right\}
$$

be the bottom of the $L^{2}$-spectrum of the Laplace-Beltrami operator $\Delta=$ $\operatorname{div} \nabla$ on a manifold $N$ (see Chapter I of [15]). I.e. $\lambda_{1}(N)$ is the smallest value of $\lambda$ such that $(\Delta+\lambda) f=0$ admits a solution $f \in L^{2}(N)$. Set $\lambda_{1}=\lambda_{1}(M)$. Denote by $\lambda_{1}^{\text {ess }}=\sup _{K}\left\{\lambda_{1}(M \backslash K)\right\}$ where $K$ ranges over all compact subsets of $M$. Then $\lambda_{1}^{\text {ess }}$ is the infimum for the continuous spectrum or discrete eigenvalues with infinite multiplicity [13].

Cheeger's isoperimetric constant for $M$ is

$$
c_{M}=\inf _{N} \frac{\operatorname{Vol}(\partial N)}{\operatorname{Vol}(N)}
$$

where the infimum is taken over all compact $n$-dimensional submanifolds of $M$ with smooth boundary. The significance of $c_{M}$ is given by Cheeger's inequality [16] which says $\frac{c_{M}^{2}}{4} \leq \lambda_{1}$. Combining this with Corollary 3 of Brooks [13] we obtain,

Theorem. For any complete simply connected negatively curved manifold

$$
\frac{c_{M}^{2}}{4} \leq \lambda_{1} \leq \lambda_{1}^{e s s} \leq \frac{\mathrm{h}^{2}}{4} .
$$


In our case we show,

Proposition 2.14. For any NCHS, $M$, we have $\frac{c_{M}^{2}}{4}=\lambda_{1}=\lambda_{1}^{e s s}=\frac{\mathrm{h}^{2}}{4}$.

Proof. We first remark that for any fixed point $o \in M$, the Laplacian of the function $r=r(x)=d(x, o)$ is given by

$$
\Delta r=\frac{\langle\nabla r(x), \nabla \mathrm{V}(o, x)\rangle}{\mathrm{V}(o, x)}=\frac{\left.\frac{\partial}{\partial t}\right|_{t=r} \mathrm{~V}(v, t)}{\mathrm{V}(v, r)},
$$

where $v=v_{o, x}$ (defined toward the beginning of this section). Using equations 2.2 and 2.4 we may rewrite the above as

$$
\begin{aligned}
\Delta r & =\frac{(\operatorname{det} A(v, r))^{\prime}}{\operatorname{det} A(v, r)}=\operatorname{tr} A^{\prime}(v, r) A^{-1}(v, r) \\
& =\operatorname{tr} U\left(-g^{r} v\right)\left(I-E \Lambda^{-1}\right)^{-1}+U\left(g^{r} v\right)\left(\Lambda E^{-1}-I\right)^{-1} .
\end{aligned}
$$

By Theorem 2.3, the limit as $r \rightarrow \infty$ of the last expression is $\mathrm{h}$, uniformly in $v \in S M \backslash \mathcal{W}^{s}\left(v_{\mathbf{a}}\right)$. For each $\epsilon>0$, let $N_{\epsilon} \subset M$ be a choice of submanifold in Cheeger's isoperimetric inequality satisfying $\frac{\operatorname{Vol}\left(\partial N_{\epsilon}\right)}{\operatorname{Vol}\left(N_{\epsilon}\right)}=c_{M}+\epsilon$. For any fixed $\epsilon>0$ and any $\delta>0$ since $N_{\epsilon}$ is bounded then by the above computation we may choose a basepoint $o=v_{\mathbf{a}}(t)$ for some sufficiently large $t$ such that we can guarantee that $\Delta r(x, o) \geq \mathrm{h}-\delta$ for all $x \in N_{\epsilon}$. Also, we remark that $|\nabla r|=1$. Hence if $*$ denotes the Hodge operator, Stokes theorem says:

$$
\operatorname{Vol}\left(\partial N_{\epsilon}\right) \geq \int_{\partial N_{\epsilon}} * d r=\int_{N_{\epsilon}} * \Delta r \geq(\mathrm{h}-\delta) \operatorname{Vol}\left(N_{\epsilon}\right)
$$

Thus $c_{M}+\epsilon \geq \mathrm{h}-\delta$. Since $\epsilon$ and $\delta$ were arbitrary, we obtain $c_{M} \geq \mathrm{h}$.

Cheeger's inequality [16] states that $\frac{c_{M}^{2}}{4} \leq \lambda_{1}$. Finally, by Corollary 3 of Brooks [13], $\lambda_{1} \leq \lambda_{1}^{\text {ess }} \leq \frac{\mathrm{h}^{2}}{4}$. Putting these inequalities together we see they are all equalities.

Remark 2.15. We remark that the above statement is in contrast to the cocompact case where Ledrappier [39] showed $\lambda_{1}=\frac{\mathrm{h}^{2}}{4}$ iff $M$ is asymptotically harmonic (and hence symmetric). On the other hand, NCHS's need not be asymptotically harmonic (see Section 7 ). 


\section{Three Important Classes of Measures on $\partial M$ and $S M$.}

We will need to know how to differentiate measures. Consider a separable metric space $X$ with metric $\rho$ which is a countable union of directionally limited sets (see section 2.8.9 of Federer [19] for a definition). In Section 2.8.9 of [19] Federer shows that any Riemannian manifold satisfies this condition. Also, $\partial M$ equipped with the visual metric from $S_{x} M$ defined by $\angle_{x}(v(\infty), w(\infty))=\langle v, w\rangle_{x}$ is directionally limited since it is inherited from the metric on the tangent sphere.

Let $B_{\rho}(x, r)$ denote the open ball in $X$ of radius $r$ about $x$. For two locally finite Borel measures $\psi$ and $\phi$ on $X$, define the quantities

$$
\underline{D}(\psi, \phi, x)=\liminf _{r \rightarrow 0} \frac{\psi\left(B_{\rho}(x, r)\right)}{\phi\left(B_{\rho}(x, r)\right)},
$$

and

$$
\bar{D}(\psi, \phi, x)=\limsup _{r \rightarrow 0} \frac{\psi\left(B_{\rho}(x, r)\right)}{\phi\left(B_{\rho}(x, r)\right)} .
$$

We define the derivative of $\psi$ with respect to $\phi$ to be the limit $\frac{d \psi}{d \phi}(x)=$ $\underline{D}(\psi, \phi, x)=\bar{D}(\psi, \phi, x)$ whenever it exists. Theorems 2.8 .18 and 2.9.5 of [19] together imply that $\frac{d \psi}{d \phi}(x)$ exists and is finite for $\phi$-almost every $x \in X$. Theorem 2.9.15 of [19] states that $\psi$ is absolutely continuous with respect to $\phi$ if and only if $\underline{D}(\psi, \phi, x)<\infty$ for $\psi$-almost every $x \in X$ (see also Theorem 2.12 of [40] for a simpler description in the $\mathbb{R}^{n}$ case). Lastly, Theorem 2.9.7 of [19] shows that in the case of absolute continuity, $\frac{d \psi}{d \phi}(x)$ coincides with the usual Radon-Nikodym derivative $\phi$ almost everywhere. In particular, these theorems show that if $\frac{d \psi}{d \phi}(x)$ exists and is positive $\phi$ and $\psi$ almost everywhere then the two measures are mutually absolutely continuous with Radon-Nikodym derivative $\frac{d \psi}{d \phi}(x)$.

We now turn our attention to measures on $\partial M$. If we project the Lebesgue measures $\lambda_{x}$ on $S_{x} M$ to $\partial M$ under the homeomorphism which takes $v$ to its corresponding asymptotic class then we obtain what are called the visual measures which we will (by abuse of notation) also denote as $\lambda_{x}$. This is an example of a system $P_{x}$ of G-equivariant measures on $\partial M$, i.e., for $g \in \mathrm{G}, g_{*} P_{x}=P_{g x}$. There are two other important measures on $\partial M$ we want to consider, but first we shall prove a useful proposition about such systems of measures.

Proposition 3.1. For any two G-equivariant systems of finite positive Borel measures $P_{x}$ and $Q_{x}$ on $\partial M$ which do not have atoms at $v_{\mathbf{a}}(\infty)$, if the 
transitional probabilities $\frac{d P_{x}}{d P_{y}}(\xi)$ and $\frac{d Q_{x}}{d Q_{y}}(\xi)$ coincide for all $x, y \in M$ and all $\xi \in \partial M$ then $P_{x}$ and $Q_{x}$ coincide as measures up to a scalar constant.

Proof. By Theorem 7.8 of [21] (c.f. Theorem V.5.3 of [31]), the measures $P_{x}$ and $Q_{x}$ are Radon measures since they are finite Borel measures and $\partial M$ is locally compact and Hausdorff. We may equip $\partial M$ with the visual metric for any $x \in M$ defined by $\angle_{x}(v(\infty), w(\infty))=\langle v, w\rangle_{x}$ which has $r$ balls $B_{x}(\xi, r)=\left\{\eta \in \partial M \mid \angle_{x}(\xi, \eta)<r\right\}$ for $\xi \in \partial M$ and $r>0$. From the discussion at the beginning of the section, we have that $\frac{d P_{x}}{d Q_{x}}(\xi)$ exists for $Q_{x}$ almost every $\xi \in \partial M$. In particular, the measures are nonzero so $\frac{d P_{x}}{d Q_{x}}(\xi)$ exists and is positive for some fixed $x$ and $\xi \neq v_{\mathbf{a}}(\infty)$. Since the Radon-Nikodym derivatives $\frac{d P_{x}}{d P_{y}}(\xi)$ and $\frac{d Q_{x}}{d Q_{y}}(\xi)$ exist and coincide we have that $\frac{d P_{x}}{d Q_{x}}(\xi)=\frac{d P_{x}}{d Q_{x}}(\xi) \frac{d P_{y}}{d P_{x}}(\xi) \frac{d Q_{x}}{d Q_{y}}(\xi)=\frac{d P_{y}}{d d Q_{y}}(\xi)$ for every $y \in M$. Then by Gequivariance of $P_{x}$ and $Q_{x}$ and the relation $g B_{x}(\xi, r)=B_{g x}(g \xi, r)$ we obtain that $\frac{d P_{g x}}{d Q_{g x}}(\xi)=\frac{d P_{x}}{d Q_{x}}\left(g^{-1} \xi\right)$ for all $g \in \mathrm{G}$. Setting $y=g x$ we can put this together to get $\frac{d P_{x}}{d Q_{x}}(\xi)=\frac{d P_{x}}{d Q_{x}}\left(g^{-1} \xi\right)$.

Since $G$ is transitive on $\partial M \backslash v_{\mathbf{a}}(\infty)$ we have $\frac{d P_{x}}{d Q_{x}}(\eta)=\frac{d P_{x}}{d Q_{x}}(\xi)$ for all $x \in M$ and $\eta \in \partial M \backslash v_{\mathbf{a}}(\infty)$. By hypothesis $v_{\mathbf{a}}(\infty)$ has measure 0 for both $P_{x}$ and $Q_{x}$ so then we conclude by our earlier discussion that $P_{x}$ and $Q_{x}$ are in the same measure class. The Radon-Nikodym theorem implies that $P_{x}$ and $Q_{x}$ are scalar multiples (independent of $x$ ) for all $x \in M$.

In direct analogy with the co-compact case, we may define the PattersonSullivan measures on $\partial M$ as follows.

\subsection{Patterson-Sullivan Measures.}

In what follows we denote the left-invariant Haar measure on $\mathrm{G}$ by $m$.

Define the following smooth function on $M \times M$

$$
g_{s}(x, y)=\int_{\mathrm{G}} e^{-s d(x, g y)} d m(g)
$$

Notice that by definition $g_{s}$ is G-invariant and hence constant in the first factor, i.e. $g_{s}(h x, y)=g_{s}(x, y)$ for $h \in \mathrm{G}$. Also if

$$
\Delta_{\mathrm{G}}(g) \stackrel{\text { def }}{=} \operatorname{det} \operatorname{Ad}\left(g^{-1}\right)
$$

is the modular function for $g \in \mathrm{G}$, then we have the relationship

$$
g_{s}(x, y)=\Delta_{\mathrm{G}}(h) g_{s}(z, h y) \quad \forall x, y, z \in M \quad \text { and } \forall h \in \mathrm{G} .
$$


We define the critical exponent $\delta$ to be the value of $s$ for which $g_{s}(x, y)$ diverges for all $s<\delta$ and converges for all $s>\delta$. Since $G$ has bounded exponential growth $\delta$ exists and is positive. We now show that in our case $g_{\delta}$ diverges. This will turn out to simplify the definition of the PattersonSullivan measures (see [42] and [20] for other, more general situations).

As a straightforward consequence of Corollary 2.7 we have,

Lemma 3.2. The function $(s-\mathrm{h}) g_{s}(o, o)-C_{M a r}$ goes to 0 as $s \rightarrow \mathrm{h}^{+}$, where $C_{M a r}$ is the Margulis constant. In particular, $\delta=\mathrm{h}$ and $g_{\mathrm{h}}$ diverges.

Consider the finite Borel measures on $M$ defined by

$$
\mu_{x}^{s}(B) \stackrel{\text { def }}{=} \frac{1}{g_{s}(o, o)} \int_{B} e^{-s d(x, g \cdot o)} d m(g) .
$$

Since $g_{s}(o, o)=g_{s}(x, o)$ for all $x \in \mathrm{G}$ we see that the $\mu_{x}$ are probability measures.

Definition 3.3. For each $x \in M$, a Patterson-Sullivan measure $\mu_{x}$ is any finite Borel measure on $M \cup \partial M$ obtained as a weak $\operatorname{limit}_{s_{i} \rightarrow \mathrm{h}^{+}} \mu_{x}^{s_{i}}$ in the space of Borel probability measures on $M$ compactified by $\partial M$, for some sequence $s_{i} \rightarrow \mathrm{h}^{+}$.

Note that weak limits exist since the space of such measures is weakly compact. Also, $\mu_{x}$ are in fact supported only on $\partial M$ since $g_{s}(o, o)$ diverges as $s \rightarrow \mathrm{h}$. We will now show that $\mu_{x}$ is independent of the choice of weak limit. If $B \subset \partial M$ is any Borel set, and $B_{x} \subset S_{x} M$ is the corresponding set under the projection to the unit tangent sphere, then

Lemma 3.4. For any set $B \subset \partial M$,

$$
\mu_{x}(B)=\frac{1}{C_{M a r}} \int_{B_{x}} \lim _{t \rightarrow \infty} e^{-h t} V(v, t) d \lambda_{x}(v)
$$

where $C_{\mathrm{Mar}}$ is the Margulis constant. Furthermore, the $\mu_{x}$ are non-atomic.

Proof. From Lemma 3.2 we found that $g_{s}(o, o)=\frac{1}{s-\mathrm{h}}\left(C_{\mathrm{Mar}}+M(s)\right)$ for some function $M(s)$ decaying to 0 as $s \rightarrow \mathrm{h}^{+}$. Using this we compute

$$
\begin{aligned}
\mu_{x}(B)=\lim _{s \rightarrow \mathrm{h}^{+}} \frac{(s-\mathrm{h})}{C_{\mathrm{Mar}}+M(s)} & \int_{0}^{\infty} \int_{B_{x}} e^{-s t} \mathrm{~V}(v, t) d \lambda_{x}(v) \mathrm{dt}= \\
& \lim _{s \rightarrow \mathrm{h}^{+}} \frac{(s-\mathrm{h})}{C_{\mathrm{Mar}}} \int_{0}^{\infty} \int_{B_{x}} e^{-s t} \mathrm{~V}(v, t) d \lambda_{x}(v) \mathrm{dt} .
\end{aligned}
$$


Since the integrand is bounded everywhere and we are multiplying by $(s-\mathrm{h})$, only the tail of the integral with respect to $t$ will contribute anything as $s$ converges to h. So by a change of variables $t \rightarrow \frac{r}{s-\mathrm{h}}$ we obtain,

$$
\begin{aligned}
\mu_{x}(B)=\lim _{s \rightarrow \mathrm{h}^{+}} \frac{(s-\mathrm{h})}{C_{\mathrm{Mar}}} \int_{0}^{\infty} \int_{B_{x}} e^{-(s-\mathrm{h}) t} \mathrm{~V}(v, t) d \lambda_{x}(v) e^{-\mathrm{h} t} \mathrm{dt}= \\
\lim _{s \rightarrow \mathrm{h}^{+}} \frac{1}{C_{\mathrm{Mar}}} \int_{0}^{\infty} \int_{B_{x}} e^{-r} \mathrm{~V}\left(v, \frac{r}{s-\mathrm{h}}\right) e^{-\frac{\mathrm{h}}{s-\mathrm{h}} r} d \lambda_{x}(v) \mathrm{dr} .
\end{aligned}
$$

Finally, by Theorem 2.3 the integrand $\mathrm{V}\left(v, \frac{r}{s-\mathrm{h}}\right) e^{-\frac{\mathrm{h}}{s-\mathrm{h}} r}$ is everywhere bounded and converges (for $r>0$ ) as $s \rightarrow \mathrm{h}^{+}$uniformly on compact subsets of $S_{x} M \backslash \mathcal{W}^{s}\left(v_{\mathbf{a}}\right)$. Hence, by dominated convergence we may take the limit inside and integrate to obtain the stated result.

Lastly, $\mu_{x}$ has no atoms since the integrand in the statement is always bounded and $\lambda_{x}$ is non-atomic.

Let $B_{\epsilon}=B_{o}(\xi, \epsilon) \subset \partial M$ be the $\epsilon$-ball in the visual metric from the basepoint $o \in M$, and let $C H B_{\epsilon}$ be its geodesic convex hull. Then

$$
\begin{aligned}
\frac{d \mu_{x}}{d \mu_{y}}(\zeta) & =\lim _{\epsilon \rightarrow 0} \frac{\mu_{x}\left(B_{x}(\zeta, r)\right)}{\mu_{y}\left(B_{x}(\zeta, r)\right)} \\
& =\lim _{\epsilon \rightarrow 0} \lim _{s \rightarrow \mathrm{h}^{+}} \frac{\mu_{x}^{s}\left(B_{\epsilon}\right)}{\mu_{y}^{s}\left(B_{\epsilon}\right)} \\
& =\lim _{\epsilon \rightarrow 0} \lim _{s \rightarrow \mathrm{h}^{+}} \frac{\int_{C H B_{\epsilon}} e^{-s d(x, g \cdot o)} d m(g)}{\int_{C H B_{\epsilon}} e^{-s d(y, g \cdot o)} d m(g)} \\
& =\lim _{\epsilon \rightarrow 0} \lim _{s \rightarrow \mathrm{h}^{+}} \frac{\int_{C H B_{\epsilon}} e^{s(d(y, g \cdot o)-d(x, g \cdot o))} e^{-s d(y, g \cdot o)} d m(g)}{\int_{C H B_{\epsilon}} e^{-s d(y, g \cdot o)} d m(g)} \\
& =e^{\mathrm{h} \rho_{x, \xi}(y)} .
\end{aligned}
$$

The last equality holds because the quantity $e^{s(d(y, z)-d(x, z))}$ is bounded for $z \in C H B_{\epsilon}$ and converges as $\epsilon \rightarrow 0$ to $e^{\mathrm{h} \rho_{x, \xi}(y)}$ where $\rho_{x, \xi}(y)$ is the Busemann function.

Remark 3.5. The Patterson-Sullivan measures are $G$ equivariant (i.e. $\left.\mu_{g x}(g B)=\mu_{x}(B)\right)$. It suffices to show that for each $s>\mathrm{h}$ the measures $\mu_{x}^{s}$ are $\mathrm{G}$ equivariant. This in turn follows from the $\mathrm{G}$ transformation properties of $g_{s}(x, y)$ and that we integrate against a left invariant Haar measure in the definition. 


\subsection{Spherical Harmonic Measures.}

Consider the Laplace-Beltrami operator $\Delta$ on $M$. The Dirichlet problem is solvable for $M$ according to the work of Sullivan [43] and Anderson and Schoen [4]. Hence for any continuous function $f$ on $\partial M$ there is a harmonic function $u_{f}$, continuous on $M \cup \partial M$, which takes the values of $f$ on $\partial M$. The spherical harmonic measure $\nu_{x}$ for a point $x \in M$ is defined as the unique measure such that for all $f \in C^{0}(\partial M)$,

$$
u_{f}(x)=\int_{\partial M} f(\xi) d \nu_{x}(\xi) .
$$

Notice that these are probability measures since for $f=1$, the function $u_{f}=1$ is harmonic.

For $x, y \in M$ we have the Green's kernel $G(x, y)$ for the operator $\Delta$ which is a harmonic function in $x$ and $y$ separately, has an integrable pole at $y=x$, and decays to zero on the boundary. In [4], M. Anderson and R. Schoen proved that

$$
\frac{d \nu_{y}}{d \nu_{x}}(\xi)=K(x, y, \xi) \stackrel{\text { def }}{=} \lim _{z \rightarrow \xi} \frac{G(y, z)}{G(x, z)}
$$

exists for all $\xi \in \partial M . K$ is also harmonic in $y$ and $K(x, \eta, \xi)=0$ for all $\eta \neq \xi$.

Theorem 1 (and Remark 2.1) of [2] show that $K(x, y, \xi) \rightarrow \infty$ as $y \rightarrow \xi$. The harmonic representation formula then implies that $\nu_{x}$ has no atoms.

Remark 3.6. Later in Proposition 6.4 and Lemma 6.5 we will show that there is a global constant $C>0$ independent of $v \in S M$ such that

$$
\frac{1}{C}<G(v(0), v(t)) e^{-\mathrm{h} t}<C
$$

for all $t>1$. In Theorem 6.10 the limit as $t \rightarrow \infty$ is explicitly given.

The next category of measures we will look at are closely related to the harmonic measures.

\section{3. $\lambda$-Harmonic Densities.}

We will follow Ancona [3] for the general theory in this subsection which is not specific to the homogeneous case. Recall $\lambda_{1}$ is the bottom of the 
spectrum of the Laplacian. Then for $\lambda<\lambda_{1}$ we have Green's kernels $G_{\lambda}$ : $M \times M \rightarrow(0, \infty]$ for the operator $\Delta+\lambda$ satisfying

$$
G_{\lambda}(x, y)=\int_{0}^{\infty} e^{\lambda t} p_{t}(x, y) d t
$$

where $p_{t}(x, y)$ is the heat kernel, i.e. the kernel for the operator $\frac{d}{d t}-\Delta$. Hence, in the discussion above for harmonic measures, we had $G(x, y)=$ $G_{0}(x, y)$. We also have the following estimates for $\lambda^{\prime}<\lambda<\lambda_{1}$ (equations $6^{\prime}$ and 7 of [3]),

$$
\begin{aligned}
& G_{\lambda}(x, y) \leq C e^{-\beta^{\prime} d(x, y)} \quad \text { for } x, y \in M, d(x, y) \geq 1 \text {, } \\
& G_{\lambda^{\prime}}(x, y) \leq C e^{-\beta d(x, y)} G_{\lambda}(x, y) \quad \text { for } x, y \in M,
\end{aligned}
$$

where $\beta, \beta^{\prime}$ and $C$ are positive constants depending only on curvature bounds $a, b$, dimension $n, \lambda$, and $\lambda^{\prime}$.

In the case of a homogeneous space, for any fixed $y \in M$ (in the future we will take $y=o$ ) we can define, in analogy with Patterson-Sullivan measures, the function

$$
n_{\lambda}(x, y)=\int_{\mathrm{G}} G_{\lambda}(x, g y) d m(g) .
$$

Since the Ricci curvature is bounded below, a result of Yau (see [15]) states that for the left invariant Haar measure $m$ corresponding to the Riemann volume, $1=\int_{\mathrm{G}} p_{t}(x, g \cdot o) d m(g)$. Expressing this using the modular function gives $\operatorname{det} \operatorname{Ad}(h)=\int_{\mathrm{G}} p_{t}(x, g h \cdot o) d m(g)$. Hence,

$$
n_{\lambda}(x, h \cdot o)=\operatorname{det} \operatorname{Ad}(h) \int_{0}^{\infty} e^{\lambda t} d t .
$$

We then see that for $\lambda \geq 0, n_{\lambda}$ diverges, but equals $-\frac{1}{\lambda} \operatorname{det} \operatorname{Ad}(h)$ for all $\lambda<\lambda_{0}=0$. We then call $0=\lambda_{0}$ the critical value. Then for $B \subset M$ and $\lambda<0$, we define

$$
\nu_{x}^{\lambda}(B)=\frac{\int_{B} G_{\lambda}(x, g \cdot o) d m(g)}{n_{\lambda}(o, o)}=-\lambda \int_{B} G_{\lambda}(x, g \cdot o) d m(g) .
$$

For each $\lambda<0$, this forms a G-equivariant family of probability measures on $M$. Since the space of probability measures on the compactification $M \cup \partial M$ is itself weakly compact, we may take a weak limit to obtain the spherical 0 -harmonic measures,

$$
\nu_{x}^{0}=\lim _{\lambda \rightarrow 0^{-}} \nu_{x}^{\lambda} .
$$


Notice that from this definition $\nu_{x}^{0}$ is a probability measure supported on $\partial M$. Also, the G-equivariance implies that the particular weak limit chosen can be taken independent of $x$. The next proposition shows that these measures are independent of the choice of weak limit.

Proposition 3.7. For all $x \in M, \nu_{x}^{0}=\nu_{x}$.

Proof. We would like to apply Proposition 3.1 but to do so we must prove that the $\nu_{x}^{0}$ are non-atomic with the same Radon-Nikodym derivatives as the $\nu_{x}$.

First we compute the Radon-Nikodym derivatives for the $\nu_{x}^{0}$. Let $B_{\epsilon} \subset$ $\partial M$ be a sequence of balls in the cone topology converging to $\xi$ as $\epsilon \rightarrow 0$ and let $C H B_{\epsilon}$ be their geodesic convex hull. Then

$$
\begin{aligned}
& \frac{d \nu_{y}^{0}}{d \nu_{x}^{0}}(\xi)=\lim _{\epsilon \rightarrow 0} \frac{\nu_{y}^{0}\left(B_{\epsilon}\right)}{\nu_{x}^{0}\left(B_{\epsilon}\right)} \\
& =\lim _{\epsilon \rightarrow 0} \lim _{\lambda \rightarrow 0^{-}} \frac{\nu_{y}^{\lambda}\left(B_{\epsilon}\right)}{\nu_{x}^{\lambda}\left(B_{\epsilon}\right)} \\
& =\lim _{\epsilon \rightarrow 0} \lim _{\lambda \rightarrow 0^{-}} \frac{\int_{C H B_{\epsilon}} G_{\lambda}(y, z) d m(z)}{\int_{C H B_{\epsilon}} G_{\lambda}(x, z) d m(z)} \\
& =\lim _{\epsilon \rightarrow 0} \lim _{\lambda \rightarrow 0^{-}} \frac{\int_{C H B_{\epsilon}} \frac{G_{\lambda}(y, z)}{G_{\lambda}(x, z)} G_{\lambda}(x, z) d m(z)}{\int_{C H B_{\epsilon}} G_{\lambda}(x, z) d m(z)} \\
& =K(x, y, \xi) \text {. }
\end{aligned}
$$

The last equality holds because the ratio $\frac{G_{\lambda}(y, z)}{G_{\lambda}(x, z)}$ is bounded for $z \in C H B_{\epsilon}$ and converges to $K_{\lambda}(x, y, \xi)$ as $\epsilon \rightarrow 0$. Furthermore, the $\lambda$-Green's kernels $G_{\lambda}(x, y)$ converge to $G(x, y)$ as shown in [2] yielding the result.

Next, $K(x, y, \xi) \rightarrow \infty$ as $y \rightarrow \xi$. So if $\nu_{x}^{0}$ had an atom at $\xi$, the above Radon-Nikodym derivative shows that the weight of the atom for $\nu_{y}^{0}$ would grow arbitrarily large as $y \rightarrow \xi$, contradicting that it is a probability measure. Hence the $\nu_{x}^{0}$ have no atoms.

Since the $\nu_{x}^{0}$ are by construction finite Borel measures we may apply Proposition 3.1 to these two systems of probability measures to finish the proof.

Thus $\nu_{x}$ is described by 3.4, which we will use in Section 6 to prove 
Lemma 6.13 which states that for a certain constant $C$,

$$
\frac{d \nu_{x}}{d \lambda_{x}}(\xi)=\frac{1}{C} \lim _{t \rightarrow \infty} \mathrm{V}\left(v_{x, \xi}, t\right) G\left(v_{x, \xi}, t\right) .
$$

We now turn our attention to three canonical G-invariant, flow-invariant measures on $S M$ related to the above measures. We will define these in our context after Kaimanovich [35], and then explore their structure.

\subsection{The Kaimanovich Construction.}

Set $\partial^{2} M=\partial M \times \partial M \backslash\{$ diagonal $\}$. Kaimanovich [35] developed a correspondence between $\sigma$-finite Radon measures on $\partial^{2} M$ (space of geodesics) and flow invariant measures on $S M$. In fact, $S M$ is homeomorphic to $\partial^{2} M \times \mathbb{R}$ by the map $v \mapsto\left(v(\infty), v(-\infty), \rho_{v}(o)\right)$. Given any symmetric function $\phi(x, y)$ on $M \times M$ and any system of equivalent measures $\tau_{x}$ on $\partial M$ satisfying

$$
\frac{d \tau_{x}}{d \tau_{y}}(\xi)=\lim _{z \rightarrow \xi} \frac{\phi(x, z)}{\phi(y, z)}
$$

then we can form a flow invariant measure $\tau$ on $S M$, by formally setting

$$
d \tau(v)=f_{x}(v(\infty), v(-\infty)) d \tau_{x}(v(\infty)) d \tau_{x}(v(-\infty)) d t
$$

where

$$
f_{x}(\xi, \eta)=\lim _{\substack{y_{1} \rightarrow \xi \\ y_{2} \rightarrow \eta}} \frac{\phi\left(y_{1}, x\right) \phi\left(x, y_{2}\right)}{\phi\left(y_{1}, y_{2}\right)} .
$$

This definition of the measure $\tau$ is independent of the choice of $x$, whenever $f_{x}$ exists.

\subsection{Liouville Measure.}

Liouville measure $\lambda$ is defined by $d \lambda(v) \stackrel{\text { def }}{=} d \lambda_{x}(v) d m(x)$ where $m$ is the unique left invariant Haar measure coinciding with the Riemannian volume, $x$ is the footpoint of $v$, and $\lambda_{x}$ is the Riemannian volume on the unit tangent sphere $S_{x} M$. This coincides with the Riemannian volume on $S M$ endowed with the Sasaki metric. This is G-invariant since the Haar measure $m$ is G-invariant and the $\lambda_{x}$ are G-equivariant. It is flow-invariant since it can also be described as the volume form $\theta \wedge d \theta^{n}$ where $\theta$ is the pullback of the canonical contact 1 -form on the cotangent bundle. 
The Liouville measure corresponds to the measure $\tau$ in the Kaimanovich construction formed by setting $\phi(x, y)=\mathrm{V}(x, y)$ and $\tau_{x}=\lambda_{x}$. Kaimanovich attributes this to Otal (see [35]). Notice that $\phi(x, y)=\mathrm{V}(v, t)$ if $v$ and $t$ are chosen so that $v(0)=x$ and $v(t)=y$. See Lemma 6.12 of [11] for an explanation of the symmetry of $\phi(x, y)$ for this case. In particular, $\frac{d \lambda_{y}}{d \lambda_{x}}(\xi)=$ $\lim _{z \rightarrow \xi} \frac{\phi(x, z)}{\phi(y, z)}$. We will supply a direct proof of this in Proposition 5.1.

\subsection{Harmonic Measure.}

The harmonic measure $\nu$ is defined to be

$$
d \nu(\eta, \zeta, t) \stackrel{\text { def }}{=} G_{x}(\eta, \zeta) d \nu_{x}(\eta) d \nu_{x}(\zeta) d t
$$

where $\nu_{x}$ are the harmonic measures at infinity, and $G_{x}$ is called the Green's cocycle and is defined by

$$
G_{x}(\eta, \zeta) \stackrel{\text { def }}{=} \lim _{\substack{y_{1} \rightarrow \eta \\ y_{2} \rightarrow \zeta}} \frac{G\left(y_{1}, y_{2}\right)}{G\left(x, y_{1}\right) G\left(x, y_{2}\right)}
$$

This corresponds to taking $\tau_{x}=\nu_{x}$ and $\phi(x, y)=\frac{1}{G(x, y)}$ in the Kaimanovich construction. Note $G_{x}(\xi, \eta)$ is equivariant with respect to isometries since the Green's functions are. The boundedness of $G_{x}$ follows from Theorem 5 of [2]. Also, this definition of $\nu$ does not depend on the choice of $x$ since it is a simple check that $K(x, y, \xi) K(x, y, \eta) G_{y}(\xi, \eta)=G_{x}(\xi, \eta)$. Hence, the continuity of $G_{x}(\xi, \eta)$ follows from the continuity of $K$. To check Ginvariance we simply take $g \in \mathrm{G}$ and compute

$$
\begin{aligned}
\frac{d \nu(\xi, \eta, t)}{d \nu(g \xi, g \eta, t)} & =\frac{G_{x}(\xi, \eta) d \nu_{x}(\xi) d \nu_{x}(\eta)}{G_{x}(g \xi, g \eta) d \nu_{x}(g \xi) d \nu_{x}(g \eta)} \\
& =\frac{G_{x}(\xi, \eta) d \nu_{x}(\xi) d \nu_{x}(\eta)}{G_{g^{-1} x}(\xi, \eta) d \nu_{g^{-1} x}(\xi) d \nu_{g^{-1} x}(\eta)} \\
& =1 .
\end{aligned}
$$

We can also do the exact same construction replacing $\mathrm{G}$ and $\nu$ everywhere by $G_{0}$ and $\nu^{0}$ to produce the corresponding $\lambda_{0}$-harmonic measure. However, by Proposition 3.7 these coincide in the homogeneous case.

\subsection{Bowen-Margulis Measure.}

The Bowen-Margulis measure $\mu$ is defined to be

$$
d \mu(\eta, \zeta, t) \stackrel{\text { def }}{=} e^{-\mathrm{h} \beta_{x}(\eta, \zeta)} d \mu_{x}(\eta) d \mu_{x}(\zeta) d t .
$$


Here $\beta_{x}$ is the Busemann cocycle defined by $\beta_{x}(\xi, \eta)=\rho_{x, \xi}(y)-\rho_{x, \eta}(y)$ for any $y$ on the geodesic between $\eta$ and $\zeta$. This corresponds to taking $\tau_{x}=\mu_{x}$ and $\phi(x, y)=e^{\mathrm{h} d(x, y)}$ in the Kaimanovich construction. Also, since the Busemann functions are G-equivariant it follows that $\beta_{x}$ is. As above, $\mu_{x}$ are the Patterson-Sullivan measures at infinity. This definition is independent of the choice of $x$ since

$$
\rho_{x, \xi}(y)+\rho_{x, \eta}(y)+\beta_{x}(\xi, \eta)=\beta_{y}(\xi, \eta) .
$$

Again this is by definition flow-invariant and we check G-invariance by

$$
\begin{aligned}
\frac{d \mu(\xi, \eta, t)}{d \mu(g \xi, g \eta, t)} & =\frac{e^{\beta_{x}(\xi, \eta)} d \mu_{x}(\xi) d \mu_{x}(\eta)}{e^{\beta_{x}(g \xi, g \eta)} d \mu_{x}(g \xi) d \mu_{x}(g \eta)} \\
& =\frac{e^{\beta_{x}(\xi, \eta)} d \mu_{x}(\xi) d \mu_{x}(\eta)}{e^{\beta_{g^{-1}}(\xi, \eta)} d \mu_{g^{-1} x}(\xi) d \mu_{g^{-1} x}(\eta)} \\
& =1
\end{aligned}
$$

\subsection{Harmonic Measures Along Foliations.}

For a foliation $\mathcal{F}$ of $S M$ with $C^{2}$ leaves, we let $C^{2, \mathcal{F}}$ be the space of continuous functions on $S M$ which are $C^{2}$ along the leaves of $\mathcal{F}$. In analogy with the case of foliations of compact spaces (see [23]) we shall call a measure $\tau$ of $S M$ harmonic with respect to $\mathcal{F}$ if

$$
0=\int_{S M} \Delta^{\mathcal{F}} f d \tau
$$

for all $f \in C^{2, \mathcal{F}}$ with $\Delta^{\mathcal{F}} f$ integrable, where $\Delta^{\mathcal{F}}$ is the Laplace-Beltrami operator along the leaves determined by a family of distinguished metrics on the leaves.

Harmonicity of the measure $\tau$ is equivalent to the property that on any flow box $E$ its corresponding system of conditional measures $\sigma_{y}$ along the plaques $E(y)$ satisfy $d \sigma_{y}(x)=h_{y}(x) d m_{y}(x)$ for $\alpha$ almost every $y$, where $m_{y}$ is the Lebesgue measure on the plaque $E(y)$ with respect to the metric induced from the leaf, and $h_{y}(x)$ is a harmonic function on $E(y)$ with respect to the Laplace-Beltrami operator on the leaf.

For the four foliations $\mathcal{W}^{s s}, \mathcal{W}^{s}, \mathcal{W}^{u}, \mathcal{W}^{s u}$ we provide the leaves with the pullback metric under the projection to $M$. We may define four measures (locally disintegrated) as

- $d \omega^{s s}(v)=d \mu_{P v}(v(\infty)) \times d m(P v)$ 
- $d \omega^{s}(v)=d \nu_{P v}(v(\infty)) \times d m(P v)$

- $d \omega^{s u}(v)=d \mu_{P v}(v(-\infty)) \times d m(P v)$

- $d \omega^{u}(v)=d \nu_{P v}(v(-\infty)) \times d m(P v)$.

We will see in Corollaries 4.2 and 4.4 that these are (not necessarily unique) harmonic measures for the corresponding foliations.

Remark 3.8. Note that since left invariant Haar measure $m$ on $M$ is unique up to scalar, every G-invariant measure on $S M$ projects to a multiple of Lebesgue measure on $M$. This is quite different from the compact case (see Conjecture 2.8.4 of Yue [50]).

\section{Integral Equations.}

In this section we will establish some integral formulas and apply them to obtain some characterizations of asymptotic harmonicity.

Note that from equations 2.3,

$$
\operatorname{tr} U\left(g^{t} v\right)=-\frac{(\operatorname{det} E(v, t))^{\prime}}{\operatorname{det} E(v, t)}=-\frac{(\operatorname{det} \Lambda(-v,-t))^{\prime}}{\operatorname{det} \Lambda(-v,-t)} .
$$

Geometrically, $\operatorname{tr} U(v)$ can be interpreted as the mean curvature of the horosphere $P \mathcal{W}^{s s}(v)$.

We similarly define

$$
\left.\tau(v) \stackrel{\text { def }}{=} \frac{d}{d t}\right|_{t=0} K(v(0), v(t), v(\infty))
$$

where $K$ is the Poisson kernel.

The visual function $r_{x}: S M \rightarrow S_{x} M$ is defined by $r_{x}(v)=\pi_{x}(v(\infty))$. Notice that $r_{g x}(d g(v))=d g\left(r_{x}(v)\right)$.

Now we begin by presenting two integral formulas proven by C. Yue in the case of compact manifolds (see Theorem 1.9.4 of [50]). We also prove a similar formula specific to NCHS's. We recall from Section 3.8 that $\Delta^{s s}$ makes sense since each horosphere is a $C^{\infty}$ manifold by Remark 2.13. Let $C_{c}^{i}$ denote $C^{i}$ functions of compact support. Let $Q$ be the trace of the $(2,1)$ tensor ad, that is for any choice of orthonormal basis $X_{i}$ of $\mathfrak{g}, Q=\operatorname{tr}$ ad $=$ $\sum_{j}\left(\operatorname{tr} \operatorname{ad}_{X_{j}}\right) X_{j}$. In what follows we will consider $Q$ as a left invariant vector field. Now we present a pair of Propositions whose integral formulas relate the measures $\mu_{x}, \nu_{x}, \omega^{s}$, and $\omega^{s s}$ to asymptotic harmonicity. 
Proposition 4.1. For all $\phi \in C_{c}^{1}(S M)$,

$$
\int_{S M}(\dot{\phi}+(\mathrm{h}-\operatorname{tr} U) \phi) d \mu_{x} d m(x)=0,
$$

and if $\phi \in C_{c}^{2}(S M)$ then

$$
\int_{S M} \Delta^{s s} \phi d \mu_{x} d m(x)=0
$$

Furthermore,

$$
\int_{S_{x} M} \dot{\phi}(v)+(\mathrm{h}+\langle v, Q\rangle-\operatorname{tr} U(v)) \phi(v) d \mu_{x}(v)=0
$$

for all G-invariant $\phi \in C^{1}(S M)$.

Proof. The first two formulas follow by the same proof in [50] and [48] which work with our given hypotheses as well. For the third formula, define a vector field by

$$
Y(y) \stackrel{\text { def }}{=} \int_{S_{y} M} \phi X(v) d \mu_{y}(v)=\int_{S_{x} M} \phi\left(r_{y}(v)\right) X\left(r_{y}(v)\right) e^{-\mathrm{h} \rho_{v}(y)} d \mu_{x}(v)
$$

where $r_{x}(v)=v_{x, v(\infty)}$ is the visual function, and $X(v)$ is the geodesic spray projected to $S M$ (so $X(v)=v$ ).

We calculate that $\left.\operatorname{div}\right|_{y=x} X\left(r_{y}(v)\right)=-\operatorname{tr} U(v)$ for $v \in S_{x} M$ and $r_{y}\left(g^{t} v\right)=r_{y}(v)$. Then we have on the one hand,

$$
\begin{aligned}
\left.\operatorname{div}\right|_{y=x} Y(y) & =\left.\int_{S_{x} M} \operatorname{div}\right|_{y=x}\left(\phi\left(r_{y}(v)\right) X\left(r_{y}(v)\right) e^{-\mathrm{h} \rho_{v}(y)}\right) d \mu_{x}(v) \\
& =\int_{S_{x} M}(\dot{\phi}+(\mathrm{h}-\operatorname{tr} U(v)) \phi) d \mu_{x}(v) .
\end{aligned}
$$

However, when $\phi$ is G-invariant we notice that $Y(y)$ is a left-invariant vector field. Observe that for the orthonormal basis $\left\{X_{i}\right\}$ of $\mathfrak{g},\left\langle\left[X_{i}, Y\right], X_{i}\right\rangle=$ $\sum_{j}\left\langle Y, X_{j}\right\rangle\left\langle\left[X_{i}, X_{j}\right], X_{i}\right\rangle$. Now we can again calculate $\left.\operatorname{div}\right|_{y=x} Y(y)$. Using the formula for the connection (2.1),

$$
\left\langle\nabla_{X_{i}} Y, X_{i}\right\rangle=\frac{1}{2}\left(\left\langle\left[X_{i}, Y\right], X_{i}\right\rangle-\left\langle Y,\left[X_{i}, X_{i}\right]\right\rangle-\left\langle X_{i},\left[Y, X_{i}\right]\right\rangle\right)=\left\langle\left[X_{i}, Y\right], X_{i}\right\rangle .
$$


By the definition of the divergence we have

$$
\begin{aligned}
\operatorname{div} Y & =\sum_{i}\left\langle\nabla_{X_{i}} Y, X_{i}\right\rangle=\sum_{i}\left\langle\left[X_{i}, Y\right], X_{i}\right\rangle \\
& =\sum_{i, j}\left\langle Y, X_{j}\right\rangle\left\langle\left[X_{i}, X_{j}\right], X_{i}\right\rangle=-\left\langle Y, \sum_{j}\left(\sum_{i}\left\langle\operatorname{ad}_{X_{j}} X_{i}, X_{i}\right\rangle\right) X_{j}\right\rangle \\
& =-\langle Y, Q\rangle \\
& =-\int_{S_{x} M} \phi\langle v, Q\rangle d \mu_{x}(v) .
\end{aligned}
$$

On the other hand, we already calculated $\left.\operatorname{div}\right|_{y=x} Y(y)$ in the first statement of the lemma. We equate the two calculations to obtain the statement.

The second formula above shows that

Corollary 4.2. $d \omega^{s s}=d \mu_{x} d m(x)$ is harmonic for the $\mathcal{W}^{\text {ss }}$ foliation.

We have similar formulas for $\nu_{x}$, see Yue [50]. The proof of the third formula is entirely analogous to that in Proposition 4.1.

Proposition 4.3. For all $\phi \in C_{c}^{1}(S M)$,

$$
\int_{S M}(\dot{\phi}+(\tau-\operatorname{tr} U) \phi) d \omega^{s}=0
$$

and if $\phi \in C_{c}^{2}(S M)$ then

$$
\int_{S M} \Delta^{s} \phi d \nu_{x} d m(x)=0
$$

Furthermore

$$
\int_{S_{x} M} \dot{\phi}+(\tau+\langle v, Q\rangle-\operatorname{tr} U) \phi d \nu_{x}=0
$$

for all G-invariant $\phi \in C^{1}(S M)$.

The second formula above shows that

Corollary 4.4. $d \omega^{s}=d \nu_{x} d m(x)$ is harmonic for the $\mathcal{W}^{s}$ foliation. 


\section{Lemma 4.5.}

$$
\mathrm{h}=\int_{S_{x} M} \operatorname{tr} U(v) d \mu_{x}
$$

and

$$
\int_{S_{x} M}\langle \pm v, Q\rangle d \mu_{x}=0
$$

Proof. Recall that the $\mu_{x}$ are probability measures so we may write

$$
1=\mu_{x}\left(S_{x} M\right)=\int_{S_{x} M} d \mu_{x}=\int_{S_{o} M} e^{-\mathrm{h} \rho_{v}(x)} d \mu_{o} .
$$

Now we use the following formula for the Laplacian of the Busemann function (see [50])

$$
\Delta e^{-\mathrm{h} \rho_{x, \xi}(y)}=\left(\mathrm{h}^{2}-\mathrm{h} \operatorname{tr} U\left(v_{y, \xi}\right)\right) e^{-\mathrm{h} \rho_{x, \xi}(y)} .
$$

Taking the Laplacian of $\mu_{x}\left(S_{x} M\right)$ yields

$$
\begin{aligned}
0=\Delta \mu_{x}\left(S_{x} M\right) & =\int_{S_{o} M} \Delta e^{-\mathrm{h} \rho_{v}(x)} d \mu_{o} \\
& =\int_{S_{o} M} \mathrm{~h}(\mathrm{~h}-\operatorname{tr} U(v)) e^{-\mathrm{h} \rho_{v}(x)} d \mu_{o} \\
& =\mathrm{h}\left(\mathrm{h}-\int_{S_{x} M} \operatorname{tr} U(v) d \mu_{x}\right)
\end{aligned}
$$

which is the first statement. Using the third formula of 4.1, this also implies the second statement.

Lemma 4.6. $\omega^{\text {ss }}$ is a $g^{t}$-invariant measure if and only if $\operatorname{tr} U=\mathrm{h}$, i.e. if and only if $M$ is asymptotically harmonic. Similarly, $\omega^{s}$ is a $g^{t}$-invariant measure if and only if $\tau=\operatorname{tr} U$.

Proof. From Proposition 4.1 we have for all $\phi \in C_{c}^{1}(S M)$,

$$
0=\int_{S M}(\dot{\phi}+(\mathrm{h}-\operatorname{tr} U) \phi) \mathrm{d} \omega^{\mathrm{ss}} .
$$

Thus $\operatorname{tr} U=\mathrm{h}$ if and only if for all $\phi \in C_{c}^{1}(S M)$,

$$
\begin{aligned}
0 & =\int_{S M} \dot{\phi} \mathrm{d} \omega^{\mathrm{ss}} \\
& =\left.\frac{d}{d t}\right|_{t=0} \int_{S M} \phi\left(g^{t} v\right) \mathrm{d} \omega^{\mathrm{ss}}
\end{aligned}
$$


This happens if and only if $\omega^{s s}$ is $g^{t}$-invariant.

The $\omega^{s}$ case follows the same way from the integral formula in Proposition 4.3.

We now show that $\mu_{x}$ and $\lambda_{x}$ are in the same measure class.

Lemma 4.7. Explicitly, for all $v \in S M \backslash \mathcal{W}^{s}\left(v_{\mathbf{a}}\right)$ and $x \in M$,

$$
\frac{d \mu_{x}}{d \lambda_{x}}(v)=\lim _{t \rightarrow \infty} \frac{\mathrm{V}(v, t) e^{-\mathrm{h} t}}{C_{M a r}},
$$

where the constant $C_{M a r}$ is the Margulis function. In particular, $\mu_{x}$ and $\lambda_{x}$ are absolutely continuous with each other.

Proof. If $B_{\epsilon}^{x} \subset S_{x} M$ is an $\epsilon$ neighborhood of $v$ then we may compute using the definition of the measures (recall 3.4),

$$
\begin{aligned}
\frac{d \mu_{x}}{d \lambda_{x}}(v) & =\lim _{\epsilon \rightarrow 0} \frac{\int_{B_{\epsilon}^{x}} \lim _{t \rightarrow \infty} e^{-\mathrm{h} t} \mathrm{~V}(w, t) d \lambda_{x}(w)}{C_{\mathrm{Mar}} \lambda_{x}\left(B_{\epsilon}^{x}\right)} \\
& =\lim _{t \rightarrow \infty} \frac{\mathrm{V}(v, t) e^{-\mathrm{h} t}}{C_{\mathrm{Mar}}} .
\end{aligned}
$$

The last equality only holds when $v \notin \mathcal{W}^{s}\left(v_{\mathbf{a}}\right)$ where it follows by continuity of the integrand. Notice that since $v_{\mathbf{a}}(\infty)$ is not an atom for either measure, $\mu_{x}$ and $\lambda_{x}$ are in the same measure class.

Recall the flip map $F: S M \rightarrow S M$ given by $F(v)=-v$. A measure $\eta$ on $S M$ (or on a tangent sphere $S_{x} M$ ) is said to be fip invariant if $F_{*} \eta=\eta$.

Corollary 4.8. The measure $\mu_{x}$ is absolutely continuous with its fip. Explicitly, for $v \in S_{x} M \backslash \mathcal{W}^{s}\left(v_{\mathbf{a}}\right)$,

$$
\frac{d \mu_{x}}{d F_{*} \mu_{x}}(v)=\lim _{t \rightarrow \infty} \frac{\mathrm{V}(v, t)}{\mathrm{V}(-v, t)}
$$

Proof. Since $\lambda_{x}$ is flip invariant, by the previous lemma we have the formula $\frac{d \mu_{x}}{d F_{*} \mu_{x}}(v)=\frac{d F_{x} \lambda_{x}}{d F_{*} \mu_{x}}(v) \frac{d \mu_{x}}{d \lambda_{x}}(v)=\frac{d \lambda_{x}}{d \mu_{x}}(-v) \frac{d \mu_{x}}{d \lambda_{x}}(v)=\lim _{t \rightarrow \infty} \frac{\mathrm{V}(v, t)}{\mathrm{V}(-v, t)}$.

Next we characterize asymptotic harmonicity in terms of flip invariance of $\mu_{x}$ and $\operatorname{tr} U$. 
Proposition 4.9. $M$ is asymptotically harmonic iff $\mu_{x}$ and $\operatorname{tr} U$ are flip invariant.

Proof. If $M$ is asymptotically harmonic then $\operatorname{tr} U=\mathrm{h}$. Hence equations 2.3 and Remark 2.1 applied to Corollary 4.8 imply that $\mu_{x}$ is flip invariant.

Conversely, if $\frac{d \mu_{x}}{d F_{*} \mu_{x}}(v)$ is constant then since $\mu_{x}$ and $F_{*} \mu_{x}$ are probability measures it follows that $\frac{d \mu_{x}}{d F_{*} \mu_{x}}(v) \equiv 1$. From Corollary 4.8 we have that $1=$ $\lim _{t \rightarrow \infty} \frac{\operatorname{det} \Lambda(v, t)}{\operatorname{det} \Lambda(-v, t)}$. So taking the log of both sides and applying equations 2.3 we obtain

$$
\int_{0}^{\infty} \operatorname{tr} U\left(-g^{t} v\right)-\operatorname{tr} U\left(-g^{t}(-v)\right) d t=0 .
$$

Replacing $v$ by $g^{s} v$ and performing a change of variables we obtain

$$
\begin{aligned}
\int_{0}^{\infty} \operatorname{tr} U\left(-g^{t+s}\right. & v)-\operatorname{tr} U\left(-g^{t-s}(-v)\right) d t \\
& =\int_{0}^{\infty} \operatorname{tr} U\left(-g^{t+s} v\right)-\operatorname{tr} U\left(-g^{t-s}(-v)\right)+\mathrm{h}-\mathrm{h} d t \\
& =\int_{0}^{\infty} \operatorname{tr} U\left(-g^{t+s} v\right)-\mathrm{h} d t-\int_{0}^{\infty} \operatorname{tr} U\left(-g^{t-s}(-v)\right)-\mathrm{h} d t \\
& =\int_{s}^{\infty} \operatorname{tr} U\left(-g^{t} v\right)-\mathrm{h} d t-\int_{-s}^{\infty} \operatorname{tr} U\left(-g^{t}(-v)\right)-\mathrm{h} d t .
\end{aligned}
$$

Taking derivatives with respect to $s$ yields

$$
2 \mathrm{~h}-\operatorname{tr} U\left(g^{s} v\right)-\operatorname{tr} U\left(-g^{s} v\right)=0
$$

for all $s$. Hence $\operatorname{tr} U(v)+\operatorname{tr} U(-v)=2 \mathrm{~h}$. Combining this with flip invariance of $\operatorname{tr} U$ gives $\operatorname{tr} U(v)=\mathrm{h}$.

Remark 4.10. From the proof of Lemma 2.10 we have that if a continuous function $f: S M \rightarrow S M$ is $g^{t}$-invariant and G-invariant then it is constant. (We can drop the Hölder condition since by $g^{t}$-invariance we don't care about the rate at which the function decays). For another proof see [27].

This property can be restated in terms of measures.

Remark 4.11. Any two absolutely continuous, flow invariant, left-invariant measures $\eta$ and $\eta^{\prime}$ on $S M$, with continuous Radon-Nikodym derivatives coincide up to scalar multiplication since the Radon-Nikodym derivative then satisfies the conditions of Remark 4.10, and hence are constant. 
A measure $\eta$ on $S M$ (resp. $S_{x} M$ ) is said to be flip quasi-invariant if it is absolutely continuous with its flip $F_{*} \eta$. In other words, $d \eta(-v)=f(v) d \eta(v)$ for some strictly positive function $f$ on $S M\left(S_{x} M\right)$.

We observe that the proof of Lemma 2.1 in [49] does not use the specific properties of the Patterson-Sullivan and visual measures. We restate it here in its more general form.

Proposition 4.12. Suppose $\left\{P_{x}\right\}_{x \in M}$ and $\left\{Q_{x}\right\}_{x \in M}$ are two families of pairwise absolutely continuous measures on $\partial M$. If both families are flip quasi-invariant for each $x \in M$ then for all $x \in M, P_{x}$ and $Q_{x}$ are in the same measure class.

Examining the proof given in [49] and stringing the Radon-Nikodym derivatives together yields the following explicit description of $\frac{d P_{x}}{d Q_{x}}$.

Corollary 4.13. With the setting of the above proposition, if $F_{x}: \partial M \rightarrow$ $\partial M$ is the flip map about the point $x \in M$, the points $x, z \in M$ and $\xi, \eta \in \partial M$ are arbitrary, and $y$ is any point on the geodesic $\gamma_{\xi, \eta}\left(\right.$ i.e. $\left.F_{y}(\xi)=\eta\right)$ then $\frac{d P_{x}}{d Q_{x}}(\xi)$ is related to $\frac{d P_{z}}{d Q_{z}}(\eta)$ by the following

$$
\frac{d P_{x}}{d Q_{x}}(\xi)=\frac{d P_{z}}{d Q_{z}}(\eta) \frac{d Q_{z}}{d Q_{y}}(\eta) \frac{d P_{y}}{d P_{z}}(\eta) \frac{d P_{y}}{d F_{y *} P_{y}}(\xi) \frac{d Q_{y}}{d F_{y *} Q_{y}}(\eta) \frac{d P_{x}}{d P_{y}}(\xi) \frac{d Q_{y}}{d Q_{x}}(\xi)
$$

Even without asymptotic harmonicity we always have,

Lemma 4.14. $\omega^{\text {ss }}$ is in the same measure class as $\lambda$, $\mu$, and $\nu$.

Proof. Let $\tau$ be one of $\lambda, \mu, \nu$. Then by definition of each of these measures they are flip invariant on $S M$. Hence with respect to the tangent sphere foliation $S_{x} M$ by Remark 3.8 we may disintegrate $\tau$ as $d \tau=d \tau_{x} d m(x)$ where the conditional measure $\tau_{x}$ on $S_{x} M$ is flip invariant. By Propositions 4.12 and 4.8 for each $x \in M d \mu_{x}=f d \tau_{x}$ for some positive function $f$ on $S M$. Therefore $d \omega^{s s}=f d \tau_{x} d m=f d \tau$.

Note that since for any $g \in G$ we have $d \mu_{g x}(d g(v))=d \mu_{x}(v)$ and $d \tau_{g x}(d g(v))=d \tau_{x}(v)$ since $d g$ is an isometry, we get that $f$ is G-invariant.

\section{Asymptotic Harmonicity and Jacobi Fields.}

We turn our attention to other means of examining asymptotic harmonicity. 
Proposition 5.1. For $\xi \in \partial M \backslash v_{\mathbf{a}}(\infty)$,

$$
\frac{d \lambda_{x}}{d \lambda_{y}}(\xi)=e^{\mathrm{h} \rho_{x, \xi}(y)} \lim _{t \rightarrow \infty} \frac{\mathrm{V}\left(v_{y, \xi}, t\right)}{\mathrm{V}\left(v_{x, \xi}, t\right)} .
$$

Proof. By Lemma 4.7 for $v \in S M \backslash \mathcal{W}^{s}\left(v_{\mathbf{a}}\right)$ and $x \in M$, we have

$$
\frac{d \mu_{x}}{d \lambda_{x}}(\xi)=\lim _{t \rightarrow \infty} \frac{\mathrm{V}\left(v_{x, \xi}, t\right) e^{-\mathrm{h} t}}{C_{\mathrm{Mar}}},
$$

where the constant $C_{\mathrm{Mar}}$ is the Margulis function. Hence by the generalized chain rule applied to $\frac{d \lambda_{x}}{d \mu_{x}}(\xi) \frac{d \mu_{y}}{d \lambda_{y}}(\xi)$,

$$
\frac{d \lambda_{x}}{d \lambda_{y}}(\xi)=\frac{d \mu_{x}}{d \mu_{y}}(\xi) \lim _{t \rightarrow \infty} \frac{\mathrm{V}\left(v_{y, \xi}, t\right)}{\mathrm{V}\left(v_{x, \xi}, t\right)} .
$$

Corollary 5.2. If $M$ is asymptotically harmonic then $\frac{d \lambda_{x}}{d \lambda_{y}}(\xi)$ is continuous in $x, y, \xi$.

Proof. Since $\operatorname{tr} U=\mathrm{h}$, we have that $\operatorname{det} \Lambda(v, t)=e^{\mathrm{h} t}$. Hence by Remark 2.1, $\lim _{t \rightarrow \infty} \frac{V(v, t)}{V(w, t)}=\frac{\operatorname{det}(U(w)+U(-w))}{\operatorname{det}(U(v)+U(-v))}$. This is continuous in $v, w$ along with $e^{\mathrm{h} \rho_{x, \xi}(y)}$. Hence $\frac{d \lambda_{x}}{d \lambda_{y}}(\xi)$ (strictly speaking an equivalence class in $\left.L^{2}\left(\partial M, \lambda_{y}\right)\right)$ given by Lemma 5.1 has a continuous representative with $\frac{d \lambda_{x}}{d \lambda_{y}}\left(v_{\mathbf{a}}(\infty)\right)=$ $e^{\mathrm{h} \rho_{x, \xi}(y)}$. Observe that by continuity this agrees with the value had we computed it using $\frac{d \lambda_{x}}{d \lambda_{y}}\left(v_{\mathbf{a}}(\infty)\right)=\lim _{\epsilon \rightarrow 0} \frac{\lambda_{x}\left(B_{x}\left(v_{\mathbf{a}}(\infty), \epsilon\right)\right)}{\lambda_{y}\left(B_{x}\left(v_{\mathbf{a}}(\infty), \epsilon\right)\right)}$.

\subsection{Other Characterizations of Asymptotic Harmonicity.}

First, we discuss the Sturm-Liouville formula. Recall that we can split $T S M=T^{h} \oplus T^{v} \oplus T^{o}$ where $T^{o}$ is the one dimensional span of the geodesic spray, $T^{v}$ is the vertical sub-bundle (the tangent bundle to the tangent sphere foliation), and $T^{h}$ is the horizontal sub-bundle (the orthogonal complement of $T^{v} \oplus T^{o}$ in the Sasaki metric). The fibers of $T^{h}$ and $T^{v}$ over $v \in S M$ are each canonically identified with $v^{\perp}$. With these identifications the canonical symplectic 2-form $\omega$ on $T^{h} \oplus T^{v}=v^{\perp} \oplus v^{\perp}$ takes the form $\omega\left(\left(x_{1}, y_{1}\right),\left(x_{2}, y_{2}\right)\right)=\left\langle x_{1}, y_{2}\right\rangle-\left\langle x_{2}, y_{1}\right\rangle$. The following lemma is a direct consequence of the flow-invariance of $\omega$ (for example see Lemma 3.2 of [49]), 


\section{Lemma 5.3 (Sturm-Liouville).}

$$
\Lambda^{*}(v, t)\left(U\left(-g^{t} v\right)+U\left(g^{t} v\right)\right) E(v, t)=U(-v)+U(v) .
$$

Remark 5.4. $\Lambda\left(g^{t} v,-t\right)=\Lambda^{-1}(v, t)$ since $I d=\Lambda(v, t-t)$.

From these equations we obtain the following result.

Proposition 5.5. For all $v \in S M, \operatorname{tr} U$ is flip invariant if and only if $\operatorname{det}(U(v)+U(-v))$ is constant.

Proof. From equations 2.2 we may compute

$$
\left(\Lambda^{*} E\right)^{\prime}=\Lambda^{*}\left(U\left(-g^{t} v\right)-U\left(g^{t} v\right)\right) E
$$

or simply,

$$
\Lambda^{*-1}\left(\Lambda^{*} E\right)^{\prime} E^{-1}=U\left(-g^{t} v\right)-U\left(g^{t} v\right) .
$$

Taking traces of both sides yields

$$
\operatorname{tr}\left(\Lambda^{*} E\right)^{\prime}\left(\Lambda^{*} E\right)^{-1}=\operatorname{tr} U\left(-g^{t} v\right)-\operatorname{tr} U\left(g^{t} v\right) .
$$

Since $\left(\Lambda^{*} E\right)(v, t)$ is non-degenerate for all $t \in \mathbb{R}$ we may write this as

$$
\frac{\left(\operatorname{det}\left(\Lambda^{*} E\right)\right)^{\prime}}{\operatorname{det}\left(\Lambda^{*} E\right)}=\operatorname{tr} U\left(-g^{t} v\right)-\operatorname{tr} U\left(g^{t} v\right) .
$$

Now taking determinants of the Sturm-Liouville equation (Lemma 5.3), we obtain

$$
\operatorname{det}\left(\Lambda^{*} E\right)=\frac{\operatorname{det}(U(v)+U(-v))}{\operatorname{det}\left(U\left(g^{t} v\right)+U\left(-g^{t} v\right)\right)} .
$$

This with the equation above yields

$$
\frac{\left(\operatorname{det}\left(U\left(g^{t} v\right)+U\left(-g^{t} v\right)\right)\right)^{\prime}}{\operatorname{det}\left(U\left(g^{t} v\right)+U\left(-g^{t} v\right)\right)}=\operatorname{tr} U\left(g^{t} v\right)-\operatorname{tr} U\left(-g^{t} v\right) .
$$

Hence $\operatorname{det}\left(U\left(g^{t} v\right)+U\left(-g^{t} v\right)\right)$ is constant in $t$ if and only if $\operatorname{tr} U\left(g^{t} v\right)$ is flip invariant. In this case $\operatorname{det}\left(U\left(g^{t} v\right)+U\left(-g^{t} v\right)\right)$ is constant by Remark 4.10 since $\operatorname{det}\left(U\left(g^{t} v\right)+U\left(-g^{t} v\right)\right)$ is continuous, which completes the Proposition. 
Remark 5.6. The above proof works on any complete manifold $M$ without conjugate points (see [14]) to show that $\operatorname{tr} U$ is flip invariant if and only if $\operatorname{det}(U(v)+U(-v))$ is flow invariant. If furthermore, $M$ covers a manifold with an ergodic geodesic flow then $\operatorname{det}(U(v)+U(-v))$ is also constant if it is flow invariant.

Corollary 5.7. We have

$$
\int_{-\infty}^{\infty} \operatorname{tr} U\left(g^{t} v\right)-\operatorname{tr} U\left(-g^{t} v\right) d t=0
$$

and if $C_{v_{\mathbf{a}}}=\operatorname{det}\left(U\left(v_{\mathbf{a}}\right)+U\left(-v_{\mathbf{a}}\right)\right)^{-1}$, then we have

$$
\lim _{t \rightarrow \infty} e^{-\mathrm{h} t} \mathrm{~V}(v, t)=C_{v_{\mathbf{a}}} e^{\int_{0}^{\infty} \operatorname{tr} U\left(g^{t} v\right)-\mathrm{h} d t} .
$$

Proof. From 5.1 in the proof of the above Lemma, for any $s$ and $t$ we obtain the relation

$$
\operatorname{det}\left(U\left(g^{t} v\right)+U\left(-g^{t} v\right)\right)=\operatorname{det}\left(U\left(g^{s} v\right)+U\left(-g^{s} v\right)\right) e^{\int_{s}^{t} \operatorname{tr} U\left(g^{r} v\right)-\operatorname{tr} U\left(-g^{r} v\right) d r} .
$$

In particular,

$$
\lim _{t \rightarrow \infty} \operatorname{det}\left(U\left(g^{t} v\right)+U\left(-g^{t} v\right)\right)=\operatorname{det}(U(v)+U(-v)) e^{\int_{0}^{\infty} \operatorname{tr} U\left(g^{r} v\right)-\operatorname{tr} U\left(-g^{r} v\right) d r}
$$

exists and is continuous for $v \in S M \backslash \mathcal{W}^{s}\left(v_{\mathbf{a}}\right)$ by Corollary 2.11 since $\operatorname{tr} U$ is constant on $\pm \mathcal{W}^{s}\left(v_{\mathbf{a}}\right)$.

The continuity of $U(v)$ on $S M$ for spaces of bounded negative curvature originally due to Eberlein, may be found in [30]. It follows that $\operatorname{det}\left(U\left(g^{s} v\right)+U\left(-g^{s} v\right)\right)$ is continuous and G-invariant, and uniformly continuous because it is determined by its values on the compact set $S_{x} M$. Hence $\lim _{t \rightarrow \infty} \operatorname{det}\left(U\left(g^{t} v\right)+U\left(-g^{t} v\right)\right)$ is constant on $\mathcal{W}^{s}(v)$ since it is flow-invariant. By G-invariance it is constant of $S M \backslash \mathcal{W}^{s}\left(v_{\mathbf{a}}\right)$. However, by Lemma 2.8, we find that

$$
\begin{aligned}
\operatorname{det}\left(U\left(g^{t} v_{\mathbf{a}}\right)+U\left(-g^{t} v_{\mathbf{a}}\right)\right) & =\operatorname{det}\left(\left\|_{t} e^{S t} D e^{-S t}\right\|_{-t}+\left\|_{t} e^{S t}(B+S) e^{-S t}\right\|_{-t}\right) \\
& =\operatorname{det}(D+B+S)=\operatorname{det}\left(U\left(v_{\mathbf{a}}\right)+U\left(-v_{\mathbf{a}}\right)\right) .
\end{aligned}
$$

So $\lim _{t \rightarrow \infty} \operatorname{det}\left(U\left(g^{t} v\right)+U\left(-g^{t} v\right)\right)=\operatorname{det}\left(U\left(v_{\mathbf{a}}\right)+U\left(-v_{\mathbf{a}}\right)\right)$ on $S M$. Similarly, replacing $v$ by $-v$ shows $\lim _{t \rightarrow-\infty} \operatorname{det}\left(U\left(g^{t} v\right)+U\left(-g^{t} v\right)\right)=\operatorname{det}\left(U\left(v_{\mathbf{a}}\right)+\right.$ $\left.U\left(-v_{\mathbf{a}}\right)\right)$. Then

$$
\begin{aligned}
\lim _{t \rightarrow \infty} \operatorname{det}\left(U\left(g^{t} v\right)\right. & \left.+U\left(-g^{t} v\right)\right) \\
& =\lim _{s \rightarrow \infty} \operatorname{det}\left(U\left(g^{s} v\right)+U\left(-g^{s} v\right)\right) e^{\int_{-\infty}^{\infty} \operatorname{tr} U\left(g^{r} v\right)-\operatorname{tr} U\left(-g^{r} v\right) d r}
\end{aligned}
$$


which after cancelling the constants yields the first claim.

From the above we have that

$$
\operatorname{det}(U(v)+U(-v))^{-1}=C_{v_{\mathbf{a}}} e^{\int_{0}^{\infty} \operatorname{tr} U\left(g^{r} v\right)-\operatorname{tr} U\left(-g^{r} v\right) d r} .
$$

Then the second claim follows from the first claim by equations 2.3 and 2.4, which states that

$$
\lim _{t \rightarrow \infty} e^{-\mathrm{h} t} V(v, t)=\operatorname{det}(U(v)+U(-v))^{-1} e^{\int_{0}^{\infty} \operatorname{tr} U\left(-g^{t} v\right)-\mathrm{h} d t} .
$$

If $\operatorname{tr} U=\mathrm{h}$ then from the previous corollary and Lemma 4.7 we see that $\frac{d \lambda_{x}}{d \mu_{x}}$ is constant. Then since they are both probability measures $\mu_{x}$ and $\lambda_{x}$ must coincide. Conversely, if they coincide for one (and hence all) $x \in M$ then $\omega^{s s}=\lambda$ and so by Lemma $4.6 M$ is asymptotically harmonic. This shows

Corollary 5.8. $M$ is asymptotically harmonic if and only if $\lambda_{x}=\mu_{x}$ for any $x \in M$.

We will now derive yet another characterization of asymptotic harmonicity in terms of flip invariance.

Proposition 5.9. $M$ is asymptotically harmonic if and only if both $\operatorname{tr} U$ and $\operatorname{tr} U^{2}$ are flip invariant. Similarly, $M$ is symmetric if and only if $U$ is fip invariant.

Proof. Assume $\operatorname{tr} U^{2}(v)=\operatorname{tr} U^{2}(-v)$ and $\operatorname{tr} U(v)=\operatorname{tr} U(-v)$. Then after taking traces the Riccati equation applied to $g^{t} v$ and $-g^{t} v$ becomes

$$
\operatorname{tr} \dot{U}\left(g^{t} v\right)=\operatorname{tr} U^{2}\left(g^{t} v\right)+\operatorname{tr} R\left(g^{t} v\right)
$$

and

$$
\operatorname{tr} \dot{U}\left(-g^{t} v\right)=\operatorname{tr} U^{2}\left(-g^{t} v\right)+\operatorname{tr} R\left(-g^{t} v\right)
$$

respectively. Since $R\left(g^{t} v\right)=R\left(-g^{t} v\right)$ we may subtract the two equations to obtain

$$
\operatorname{tr} \dot{U}\left(g^{t} v\right)=\operatorname{tr} \dot{U}\left(-g^{t} v\right) .
$$

However, by flip invariance of $\operatorname{tr} U$ we have $\operatorname{tr} \dot{U}\left(g^{t} v\right)=-\operatorname{tr} \dot{U}\left(-g^{t} v\right)$ hence $\operatorname{tr} \dot{U}\left(g^{t} v\right)=0$. It follows that $\operatorname{tr} U$ is $g^{t}$-invariant and G-invariant, hence 
it is constant on $S M$, and equal to h. Conversely, if $M$ is asymptotically harmonic, then $\operatorname{tr} U=\mathrm{h}$ so $\operatorname{tr} U$ is flip invariant and $\operatorname{tr} \dot{U}=0$. So by the same Riccati equation we see that $\operatorname{tr} U^{2}(v)=\operatorname{tr} R(v)$, and hence $\operatorname{tr} U^{2}$ is flip invariant.

For the second statement, again using the Riccati equation we have

$$
\dot{U}\left( \pm g^{t} v\right)=U^{2}\left( \pm g^{t} v\right)+R\left( \pm g^{t} v\right) .
$$

By flip invariance of $R$ and $U, \dot{U}\left(-g^{t} v\right)=\dot{U}\left(g^{t} v\right)$. This implies that $U$ is covariant constant. Hence we read from the Riccati equation that $U^{2}=-R$. So $R$ is covariant constant, which implies $M$ is symmetric.

Proposition 5.10. For any $x \in M, \mu_{x}=\nu_{x}$ if and only if $M$ is asymptotically harmonic, in which case $\tau=\mathrm{h}$.

Proof. If $\mu_{x}=\nu_{x}$ then by subtracting the formulas from Propositions 4.1 and 4.3 we have

$$
\int_{S_{x} M}(\mathrm{~h}-\tau) \phi d \nu_{x}=0
$$

for all $\phi \in C_{c}^{1}$. Observe that $\nu_{x}$ is positive on open sets since it is the hitting probability measure on $\partial M$ for Brownian motion. This implies that $\mathrm{h}=\tau$. Also by looking at the respective Radon-Nikodym derivatives we get $K(x, y, \xi)=e^{-\mathrm{h} \rho_{x, \xi}(y)}$ so

$$
0=\Delta K=\Delta e^{-\mathrm{h} \rho}=\mathrm{h}(\mathrm{h}-\operatorname{tr} U) e^{-\mathrm{h} \rho}
$$

so $\mathrm{h}=\operatorname{tr} U$.

Conversely, if $\operatorname{tr} U$ is constant then $\mathrm{h}=\operatorname{tr} U$ (Corollary 2.9) and consequently, $\Delta e^{-\mathrm{h} \rho_{x, \xi}(y)}=0$ and so uniqueness of the Poisson Kernel implies $K(x, y, \xi)=e^{-\mathrm{h} \rho_{x, \xi}(y)}$. Hence since $\mu_{x}$ and $\nu_{x}$ satisfy the hypotheses of Proposition 3.1 we have that $\mu_{x}=\nu_{x}$.

Remark 5.11. The above proposition holds true for negatively curved compact manifolds as well. See [50].

Before we prove the equivalence of the asymptotic harmonicity and $\nu_{x}=$ $\lambda_{x}$ we develop some notions of Kaimanovich which will be used in the proof. In what follows $\mathbb{P}^{x}$ will be the Wiener measure on continuous paths $\Omega_{x}$ starting at $x$. 
Definition 5.12. The Kaimanovich entropy $\beta$ for homogeneous spaces is defined to be the quantity

$$
\beta \stackrel{\text { def }}{=} \int_{S_{x} M}\left|\nabla_{y=x} \ln K(x, y, v(\infty))\right|^{2} d \nu_{x}(v),
$$

which by G-invariance is independent of $x$ (see [34]). Similarly define for $\mathbb{P}^{x}$ almost every Brownian motion $\omega_{t}$ on $M$,

$$
\alpha \stackrel{\text { def }}{=} \lim _{t \rightarrow \infty} \frac{d\left(x, \omega_{t}\right)}{t} .
$$

For homogeneous spaces, the following Lemma is due to Kaimanovich [32]. We include a proof for completeness.

Lemma 5.13.

$$
\alpha=\int_{S_{x} M} \operatorname{tr} U(v) d \nu_{x}(v)
$$

Proof. In Theorems 1 and 2 of [41] Prat showed the transience of Brownian motion and that $\omega_{\infty} \in \partial M$ is well defined for $\mathbb{P}^{x}$-almost every $\omega_{i}$. He also showed $d\left(\omega_{t}, \omega_{t+1}\right)=o(t)$. It follows (for example [33]) that $\lim _{t \rightarrow \infty} \frac{d\left(v_{x, \omega_{\infty}}(\alpha t), \omega_{t}\right)}{t}=0$. Then $\alpha=\lim _{t \rightarrow \infty}-\frac{\rho_{x, \omega_{\infty}}\left(\omega_{t}\right)}{t}$. By the Itô Lemma (for example [41]) we have that for $\mathbb{P}^{x}$-almost every Brownian motion $\omega_{t}$ that

$$
\alpha=\lim _{t \rightarrow \infty} \frac{1}{t} \int_{0}^{t} \Delta \rho_{x, \omega_{\infty}}\left(\omega_{s}\right) d s=\lim _{t \rightarrow \infty} \frac{1}{t} \int_{0}^{t} \operatorname{tr} U\left(v_{\omega_{s}, \omega_{\infty}}\right) d s .
$$

Then consider the dynamical system in the space of paths starting from $x$,

$$
\left(T^{\tau} \omega\right)_{t}=g\left(\omega_{\tau}\right)^{-1} \omega_{\tau+t},
$$

where $g\left(\omega_{\tau}\right)^{-1}$ is the group element sending $\omega_{\tau}$ to $x$. This preserves $\mathbb{P}^{x}$ and is ergodic so by the Birkhoff ergodic theorem,

$$
\alpha=\int_{\Omega_{x}} \operatorname{tr} U\left(v_{x, \omega_{\infty}}\right) d \mathbb{P}^{x}(\omega) .
$$

However, since $\nu_{x}$ is the hitting probability measure on $\partial M$ for paths starting at $x$, we can write this integral as

$$
\alpha=\int_{\partial M} \operatorname{tr} U\left(v_{x, \xi}\right) d \nu_{x}(\xi) .
$$

Converting to the measure on the unit tangent sphere completes the lemma. 
Lemma 5.14. The Brownian escape velocity is the volume growth entropy, i.e. $\alpha=\mathrm{h}$.

Proof. Let $p: \mathrm{G} \rightarrow \mathrm{G} / \mathrm{N}=\mathrm{A}$ be the quotient projection to the abelian line. We may write a Brownian path $\omega_{t}$ in $M$ starting at the point $o$ as $n_{t} a_{t} \cdot o$ where $a_{t}=p\left(\omega_{t}\right)$ and $n_{t} \in \mathrm{N}$. In 2.4 of [7] M. Babillot proves that almost surely,

$$
\lim _{t \rightarrow \infty} \frac{d\left(o, a_{t} \cdot o\right)}{t}=\mathrm{h}
$$

(in her notation, $2 \rho(H)=\mathrm{h}$ ). She also shows in Theorem 2 of [7] that $n_{t}$ almost surely converges to some point $n_{\infty}$. Then we may compute for almost every $\omega_{t}$,

$$
\alpha=\lim _{t \rightarrow \infty} \frac{d\left(o, \omega_{t}\right)}{t}=\lim _{t \rightarrow \infty} \frac{d\left(o, n_{t} a_{t} \cdot o\right)}{t}=\lim _{t \rightarrow \infty} \frac{d\left(n_{t}^{-1} \cdot o, a_{t} \cdot o\right)}{t} .
$$

Now since $n_{t}$ converges, then for all $t>0, d\left(o, n_{t}^{-1} \cdot o\right)<C$ for some $C>0$. Then by the triangle inequality for $t>0$,

$$
\frac{d\left(o, a_{t} \cdot o\right)-C}{t} \leq \frac{d\left(n_{t}^{-1} \cdot o, a_{t} \cdot o\right)}{t} \leq \frac{d\left(o, a_{t} \cdot o\right)+C}{t} .
$$

However taking limits as $t \rightarrow \infty$ and combining with our previous estimate yields

$$
\alpha=\lim _{t \rightarrow \infty} \frac{d\left(o, a_{t} \cdot o\right)}{t}=\mathrm{h} .
$$

Proposition 5.15. For any $x \in M, \nu_{x}=\lambda_{x}$ if and only if $M$ is asymptotically harmonic.

Proof. First if $M$ is asymptotically harmonic then by Corollary $5.8, \mu_{x}=$ $\lambda_{x}$ for each $x \in M$. At the same time, by Proposition 5.10, $\nu_{x}=\mu_{x}$. Consequently $\nu_{x}=\lambda_{x}$ for all $x \in M$.

For the converse we begin by equating the Radon-Nikodym derivatives,

$$
\frac{d \nu_{x}}{d \nu_{y}}(\xi)=\frac{d \lambda_{x}}{d \lambda_{y}}(\xi)
$$

Using Proposition 5.1 and Section 3.2 we may evaluate both sides to get

$$
K(y, x, \xi)=e^{-\mathrm{h} \rho_{x, \xi}(y)} \lim _{t \rightarrow \infty} \frac{\mathrm{V}\left(v_{y, \xi}, t\right)}{\mathrm{V}\left(v_{x, \xi}, t\right)} .
$$


Setting $f(v)=\int_{0}^{\infty} \operatorname{tr} U\left(g^{t} v\right)-\mathrm{h} d t$, we have by Corollary 5.7,

$$
K(y, x, \xi)=e^{-\mathrm{h} \rho_{x, \xi}(y)+f\left(v_{y, \xi}\right)-f\left(v_{x, \xi}\right)} .
$$

Notice also that since both $K(x, y, \xi)$ and $\rho_{y, \xi}(x)$ are smooth in $x, y$ and Hölder in $\xi$, then $f\left(v_{y, \xi}\right)-f\left(v_{x, \xi}\right)$ is also. Hence we may directly compute,

$$
\begin{aligned}
\left.\frac{d}{d t}\right|_{t=0} f\left(g^{t} v\right)=\left.\frac{d}{d t}\right|_{t=0}( & \left.f\left(g^{t} v\right)-f(v)\right) \\
& =-\left.\frac{d}{d t}\right|_{t=0} \int_{0}^{t} \operatorname{tr} U\left(g^{s} v\right)-\mathrm{h} d s=\mathrm{h}-\operatorname{tr} U(v),
\end{aligned}
$$

i.e. $\tau(v)=\operatorname{tr} U(v)$.

Since $f(v)$ is bounded on $S M$ the above shows that

$$
\lim _{t \rightarrow \infty} \frac{1}{t} \log K\left(x, \omega_{t}, \omega_{\infty}\right)=\mathrm{h} \alpha=\mathrm{h}^{2}
$$

by Lemma 5.14. However, by Theorem 2(c) of [34] which is asserted for Lie groups, the expression above is simply $\beta$.

Combining this with Hölder's inequality for the probability measure $\nu_{x}$ we get (see [49] for a similar computation)

$$
\begin{aligned}
\mathrm{h}^{2}=\beta & =\int_{S_{x} M}\left|\nabla_{y=x} \ln K(x, y, v(\infty))\right|^{2} d \nu_{x}(v) \\
& \geq\left.\int_{S_{x} M}\left|\frac{d}{d t}\right|_{t=0} K(v(0), v(t), v(\infty))\right|^{2} d \nu_{x}(v) \\
& \geq\left(\int_{S_{x} M}\left|\frac{d}{d t}\right|_{t=0} K(v(0), v(t), v(\infty)) \mid d \nu_{x}(v)\right)^{2} \\
& =\left(\int_{S_{x} M} \tau(v) d \nu_{x}(v)\right)^{2} \\
& =\left(\int_{S_{x} M} \operatorname{tr} U(v) d \nu_{x}(v)\right)^{2}=\alpha^{2} .
\end{aligned}
$$

The second to last inequality above follows from equation 5.2 and the last equality by Lemma 5.13 . Now since by Lemma $5.14 \alpha=\mathrm{h}$, all of the above inequalities are equalities. Then by the equality case for Hölder's inequality we have

$$
\tau(v)=\left|\frac{d}{d t}\right|_{t=0} K(v(0), v(t), v(\infty)) \mid \equiv \mathrm{h} .
$$

However, $\operatorname{tr} U=\tau=\mathrm{h}$. 


\section{Harmonic Measures and the Remaining Cases.}

We now complete the proof of the three theorems.

For $\lambda \leq 0$, set $K_{\lambda}(v, t)=K_{\lambda}(v(0), v(t), v(\infty))$ and $G_{\lambda}(v, t)=$ $G(v(0), v(t))$. As before, we will write $G(v, t)$ for $G_{0}(v, t)$ and $K(v, t)$ for $K_{0}(v, t)$. Following Ancona [2] we shall say that an operator $\mathcal{L}$ is weakly coercive if there exists a positive $\epsilon$ such that $\mathcal{L}+\epsilon$ admits a positive superharmonic function. For instance $\Delta$, and hence $\Delta+\lambda$ are weakly coercive operators for $\lambda<\lambda_{1}$. The boundary Harnack inequalities for weakly coercive operators (Theorem 2 of [2]) states

Theorem 6.1. Let $C(v, \theta)$ be a cone in the direction $v$ and angle $\theta$ in $M$ and set $y=v(1)$. Then if $u$ and $w$ are two positive $\mathcal{L}$-harmonic functions on $C(v, \theta)$ vanishing at $C(v, \theta) \cap \partial M$, then

$$
c^{-1} \frac{u(y)}{w(y)} \leq \frac{u(x)}{w(x)} \leq c \frac{u(y)}{w(y)},
$$

for all $x \in C\left(v^{\prime}(1), \theta\right)$ and where $c$ depends only on $\mathcal{L}, M$, and $\theta$.

By replacing the boundary Harnack inequality (Theorem 5.1 of [4]) for $\Delta$ by the boundary Harnack inequality for weakly coercive operators (Theorem 2 of [2]) we may apply the same proof of Lemma 3.2 of [25] to show

Lemma 6.2. The function $\tau_{\lambda}(v)=\left.\frac{d}{d t}\right|_{t=0} K_{\lambda}(v, t)$ is Hölder continuous on $S M$.

Similarly we may obtain the following lemma by combining the proofs of Lemma 3.10 and Lemma 3.11 of [25] and using the Harnack inequalities for weakly coercive operators. Note that the proofs do not depend on compactness of the underlying manifold and otherwise apply without change to our case.

Lemma 6.3. There are constants $C_{1}, C_{2}$ such that for $t \geq 1$, and $\lambda \leq 0$,

$$
\begin{gathered}
C_{1}^{-1} \leq K_{\lambda}(v, t) K_{\lambda}(v(0), v(t), v(-\infty)) \leq C_{1} \\
C_{2}^{-1} \leq K_{\lambda}(v(0), v(t), v(-\infty)) G_{\lambda}(v, t)^{-1} \leq C_{2} .
\end{gathered}
$$

We now state a prove a Proposition about the asymptotic exponential growth rate of these quantities. 
Proposition 6.4. There are constants $C_{1}, C_{2}>0$ independent of $v \in S M$ such that

$$
\frac{1}{C_{1}}<K_{\lambda}(v, t) e^{-\tau_{\lambda}\left(v_{\mathbf{a}}\right) t}<C_{1}
$$

and for $t>1$,

$$
\frac{1}{C_{2}}<G_{\lambda}(v, t) e^{\tau_{\lambda}\left(v_{\mathbf{a}}\right) t}<C_{2} .
$$

Furthermore, as $t \rightarrow \infty$ (resp. $t \rightarrow-\infty$ ),

$$
K_{\lambda}(v, t) e^{-\tau_{\lambda}\left(v_{\mathbf{a}}\right) t}
$$

and

$$
G_{\lambda}(v, t) e^{\tau_{\lambda}\left(v_{\mathbf{a}}\right) t}
$$

converge to continuous functions on $S M \backslash \mathcal{W}^{s}\left(v_{\mathbf{a}}\right)$ (resp. $S M \backslash \mathcal{W}^{u}\left(-v_{\mathbf{a}}\right)$ ).

Proof. By definition $K_{\lambda}$ satisfies $K_{\lambda}(x, z, \xi)=K_{\lambda}(x, y, \xi) K_{\lambda}(y, z, \xi)$ for all $x, y \in M$ and $\xi \in \partial M$. In particular, for all $v \in S M, K_{\lambda}(v, t+s)=$ $K_{\lambda}\left(g^{t} v, s\right) K_{\lambda}(v, t)$. Hence $K_{\lambda}^{\prime}(v, t)=\tau_{\lambda}\left(g^{t} v\right) K_{\lambda}(v, t)$ and so $K_{\lambda}(v, t)=$ $e^{\int_{0}^{t} \tau_{\lambda}\left(g^{s} v\right) d s}$. From 6.3 we get $K_{\lambda}\left(g^{-t} v, t\right) \simeq K_{\lambda}(-v, t)$, but by G-invariance $K_{\lambda}\left(g^{-t} v_{\mathbf{a}}, t\right)=e^{\tau_{\lambda}\left(v_{\mathbf{a}}\right) t}$ so $e^{\tau_{\lambda}\left(v_{\mathbf{a}}\right) t}=K_{\lambda}\left(g^{-t} v_{\mathbf{a}}, t\right) \simeq K_{\lambda}\left(-v_{\mathbf{a}}, t\right)=e^{\tau_{\lambda}\left(-v_{\mathbf{a}}\right) t}$ and hence $\tau_{\lambda}\left(v_{\mathbf{a}}\right)=\tau_{\lambda}\left(-v_{\mathbf{a}}\right)$. Also $\tau_{\lambda}(v)$ is Hölder continuous by Lemma 6.2 .

From this point, the proof of the rest of the claims about $K_{\lambda}$ follows the proof of Theorem 2.3 after replacing $\operatorname{tr} U$ by $\tau_{\lambda}$ and $\operatorname{det} \Lambda$ by $K_{\lambda}$. The estimate on $G_{\lambda}(v, t)$ follows from the second estimate of Lemma 6.3. Since the proof of Theorem 6.2 of [4] only depends on the Harnack inequality for harmonic functions we may apply it to weakly coercive operators. We have that the function (of $y) \frac{G_{\lambda}(x, y)}{K_{\lambda}(x, y, \xi)}$ has a Hölder extension to $(M \backslash B(x, 1)) \cup$ $(\partial M \backslash\{\xi\})$. This ratio is continuous in $x \in M$, so by G-equivariance for $g \in \mathrm{G}, \frac{G_{\lambda}(x, y)}{K_{\lambda}(x, y, \xi)}=\frac{G_{\lambda}(g x, g y)}{K_{\lambda}(g x, g y, g \xi)}$ implies that it is continuous in $\xi \notin \mathcal{W}^{s}\left(-v_{\mathbf{a}}\right)$ as well. So in particular, $\lim _{t \rightarrow \infty} \frac{G_{\lambda}(v, t) e^{\tau_{\lambda}\left(v_{\mathbf{a}}\right)}}{K_{\lambda}(-v,-t) e^{\tau_{\lambda}\left(v_{\mathbf{a}}\right)}}$ is continuous. However the limit of the denominator exists and is continuous for $v \notin \mathcal{W}^{s}\left(v_{\mathbf{a}}\right)$, so the same is true of the numerator.

Let $\tau(v)=\tau_{0}(v)$. Then we have the following estimates for $\tau_{\lambda}\left(v_{\mathbf{a}}\right)$ in terms of the curvature bounds $-b^{2}$ and $-a^{2}$. 
Lemma 6.5. For any $v \in S M$ we have

$$
\mathrm{h}=\lim _{t \rightarrow \infty} \tau\left(g^{t} v\right)
$$

in particular, $\tau\left(v_{\mathbf{a}}\right)=\mathrm{h}$. Furthermore,

$$
\tau_{\lambda}\left(v_{\mathbf{a}}\right) \geq \mathrm{h}+\left(\sqrt{\left(\frac{n-1}{2}(2 b-a)\right)^{2}-\lambda}-\left(\frac{n-1}{2}(2 b-a)\right)\right) .
$$

Proof. In polar coordinates the integral 3.3 becomes,

$$
\int_{\mathrm{G}} G_{\lambda}(x, g y) d m(g)=\int_{S_{o} M} \int_{0}^{\infty} G_{\lambda}(v, t) \mathrm{V}(v, t) d t d v .
$$

By Proposition 6.4 all of the $G_{\lambda}$ are essentially exponential functions. Since the integral above converges for $\lambda<0$ and diverges for $\lambda=0$, the asymptotic exponential decay of $G(v, t)=G_{0}(v, t)$ must be that of $\mathrm{V}(v, t)^{-1}$ which is $-\mathrm{h}$. From Proposition 6.4 , it follows that the asymptotic exponential growth of $K(v, t)$ is $\mathrm{h}$ which is equivalent to the first statement of the Lemma. The second statement is an application of Theorem 4 or [3] which shows that if $\gamma=\left(\sqrt{\left(\frac{n-1}{2}(2 b-a)\right)^{2}-\lambda}-\left(\frac{n-1}{2}(2 b-a)\right)\right)$, then $G_{\lambda}(v, t) \leq C_{\epsilon} e^{-(\gamma-\epsilon) t} G(v, t)$ for any $\epsilon>0$. Since $\tau_{\lambda}\left(v_{\mathbf{a}}\right)$ exists the inequality follows.

Corollary 6.6. The Kaimanovich Entropy equals the square of the volume growth entropy, i.e. $\beta=h^{2}$.

Proof. By Theorem 2(c) of [34] $\beta=\lim _{t \rightarrow \infty} \frac{\log K\left(x, \omega_{t}, \xi\right)}{t}$ for almost every Brownian motion conditioned on $\omega_{\infty}=\xi$. However, since $d\left(\omega_{t}, x\right)=$ $\alpha t+o(t)=\mathrm{h} t+o(t)$ it follows from Proposition 6.4 and Lemma 6.5 that $\log K\left(x, \omega_{t}, \xi\right)=\mathrm{h}^{2} t+o(t)$ which proves the corollary.

The above corollary allows us to relate the bottom of the spectrum to the heat kernel in the following way.

Corollary 6.7. The bottom of the spectrum $\lambda_{1}$ is the eigenvalue for the functions $\sqrt{p(t, x, y)}$ for all $t>0$ and $x \in M$. 
Proof. For the proof we follow a computation found in Yue [50]. In [34] Kaimanovich states that the following formula for the entropy,

$$
\begin{aligned}
\beta & =\lim _{t \rightarrow \infty}-\frac{1}{t} \int_{M} p(t, x, y) \log p(t, x, y) d m(y) \\
& =\lim _{t \rightarrow \infty}-\frac{1}{t} \int_{0}^{t}\left\{\int_{M} \frac{\partial}{\partial s} p(s, x, y)(1+\log p(s, x, y)) d m(y)\right\} d s \\
& =\lim _{t \rightarrow \infty}-\frac{1}{t} \int_{0}^{t}\left\{\int_{M} \Delta_{y} p(s, x, y)(1+\log p(s, x, y)) d m(y)\right\} d s \\
& =\lim _{t \rightarrow \infty} \frac{1}{t} \int_{0}^{t}\left\{\int_{M} \frac{\|\nabla p(s, x, y)\|^{2}}{p(s, x, y)} d m(y)\right\} d s \\
& =\lim _{t \rightarrow \infty} \frac{4}{t} \int_{0}^{t}\left\{\int_{M}\|\nabla \sqrt{p(s, x, y)}\|^{2} d m(y)\right\} d s \\
\geq & 4 \lambda_{1},
\end{aligned}
$$

since $\sqrt{p(s, x, y)}$ has $L^{2}$ norm 1. However Corollary 6.6 and Proposition 2.14 show that $\beta=4 \lambda_{1}=\mathrm{h}^{2}$. Hence by continuity of $p(s, x, y)$,

$$
\int_{M}\|\nabla \sqrt{p(s, x, y)}\|^{2} d m(y)=\lambda_{1}
$$

for all $s>0$. This implies that $\Delta \sqrt{p(t, x, y)}=-\lambda_{1} \sqrt{p(t, x, y)}$ as claimed.

Remark 6.8. In the cocompact case both $\beta=\alpha \mathrm{h}, \alpha=\mathrm{h}$ and $\beta=\mathrm{h}^{2}$ are each equivalent to asymptotic harmonicity. This was proved by F. Ledrappier [38] and [36]. The proofs use the Gibbs states coming from potential theory which require an ergodic geodesic flow.

In the proof of Proposition 5.15 all we used was that $\beta \leq \mathrm{h}^{2}$ and $\tau=\operatorname{tr} U$, hence

Corollary 6.9. $M$ is asymptotically harmonic iff $\tau=\operatorname{tr} U$.

We are now in a position to present an explicit asymptotic description for $G(v, t)$.

Theorem 6.10. For $\lambda \leq 0$, we have

$$
\lim _{t \rightarrow \infty} G_{\lambda}(v, t) e^{\tau_{\lambda}\left(v_{\mathbf{a}}\right) t}=\frac{1}{q_{\lambda}} e^{\int_{0}^{\infty} \tau_{\lambda}\left(v_{\mathbf{a}}\right)-\tau_{\lambda}\left(g^{t} v\right) d t}
$$


for the constant $q_{\lambda}=\lim _{t \rightarrow \infty} G_{\lambda}\left(v_{\mathbf{a}}, t\right) e^{t \tau_{\lambda}\left(v_{\mathbf{a}}\right)}$. In particular, $G_{\lambda}(v, t) e^{\tau_{\lambda}\left(v_{\mathbf{a}}\right) t}$ converges uniformly exponentially quickly in $t$ on compact subsets of $S M \backslash$ $\mathcal{W}^{s}\left(v_{\mathbf{a}}\right)$.

Proof. By applying Lemma 6.5 and Proposition 6.4 the function

$$
q_{\lambda}(v)=\lim _{t \rightarrow \infty} G_{\lambda}(v, t) e^{t \tau_{\lambda}\left(v_{\mathbf{a}}\right)}
$$

exists and is bounded on $S M$ and continuous on $S M \backslash \mathcal{W}^{s}\left(v_{\mathbf{a}}\right)$.

By Corollary 1 of [2] there exist $C, R>0$ independent of $x$ and $y \in$ $M \backslash B(x, R)$ such that

$$
\left|\nabla_{x} G_{\lambda}(x, y)\right| \leq C G_{\lambda}(x, y) .
$$

This along with the boundary Harnack inequality implies that there exists another $C>0$ independent of $z \in B(x, 1)$ and $y \in M \backslash B(x, R)$ such that

$$
\frac{\left|G_{\lambda}(x, y)-G_{\lambda}(z, y)\right|}{d(x, z)}<C G_{\lambda}(x, y) .
$$

Written in terms of $G_{\lambda}(v, t)$ and $G_{\lambda}(w, t)$ where $v(0)=x, w(0)=z$, and $w(t)=v(t)=y$, we have

$$
\left|G_{\lambda}(v, t)-G_{\lambda}(w, t)\right|<C d(P w, P v) G_{\lambda}(v, t) .
$$

Multiplying by $e^{t \tau_{\lambda}\left(v_{\mathbf{a}}\right)}$ and taking limits in $t$ we obtain $\left|q_{\lambda}(v)-q_{\lambda}(w)\right|<$ $C d(P w, P v) q_{\lambda}(v)$. So in particular, $q_{\lambda}(v)$ is Lipschitz on $\mathcal{W}^{s}(v)$.

Now since by G-invariance $q_{\lambda}(v)$ is constant on $\mathcal{W}^{s}\left(v_{\mathbf{a}}\right)$ and $\mathcal{W}^{u}\left(-v_{\mathbf{a}}\right)$, by Hölder continuity (on each $\mathcal{W}^{s}(v)$ ) it follows that $q_{\lambda}^{+}(v)=\lim _{s \rightarrow \infty} q\left(g^{s} v\right)$ exists and is the constant $q_{\lambda}\left(-v_{\mathbf{a}}\right)$ on each $\mathcal{W}^{s}(v) \neq \mathcal{W}^{s}\left(v_{\mathbf{a}}\right)$. I.e. $q_{\lambda}(v)$ is constant on $S M \backslash \mathcal{W}^{s}\left(v_{\mathbf{a}}\right)$. By the symmetry $G_{\lambda}(v, t)=G_{\lambda}(-v,-t)$, we have $q_{\lambda}\left(-v_{\mathbf{a}}\right)=q_{\lambda}\left(v_{\mathbf{a}}\right)$. Hence, $q_{\lambda}=q_{\lambda}^{+}(v)$ is constant on $S M$ since $q_{\lambda}\left(v_{\mathbf{a}}\right)=q_{\lambda}\left(-v_{\mathbf{a}}\right)$.

The uniform convergence of $G_{\lambda}(v, t) e^{\tau_{\lambda}\left(v_{\mathbf{a}}\right) t}$ is then a direct consequence of Corollary 2.11.

Corollary 6.11. The Poisson Kernel is given by

$$
K_{\lambda}(x, y, \xi)=e^{-\tau_{\lambda}\left(v_{\mathbf{a}}\right) \rho_{x, \xi}(y)+f\left(v_{x, \xi}\right)-f\left(v_{y, \xi}\right)}
$$

where $f(v)=\int_{0}^{\infty} \tau_{\lambda}\left(g^{t} v\right)-\tau_{\lambda}\left(v_{\mathbf{a}}\right) d t$. 
Proof. First we recall from the definition of $K_{\lambda}$ we have

$$
\begin{aligned}
K_{\lambda}(x, y, \xi) & =\lim _{z \rightarrow \xi} \frac{G_{\lambda}(y, z)}{G_{\lambda}(x, z)} \\
& =\lim _{t \rightarrow \infty} \frac{G_{\lambda}\left(v_{y, \xi}, t+\rho_{x, \xi}(y)\right)}{G_{\lambda}\left(v_{x, \xi}, t\right)} \\
& =e^{-\tau_{\lambda}\left(v_{\mathbf{a}}\right)} \lim _{t \rightarrow \infty} \frac{G_{\lambda}\left(v_{y, \xi}, t+\rho_{x, \xi}(y)\right) e^{\left(t+\rho_{x, \xi}(y)\right) \tau_{\lambda}\left(v_{\mathbf{a}}\right)}}{G_{\lambda}\left(v_{x, \xi}, t\right) e^{t \tau\left(v_{\mathbf{a}}\right)}} \\
& =e^{-\tau_{\lambda}\left(v_{\mathbf{a}}\right) \rho_{x, \xi}(y)+f\left(v_{x, \xi}\right)-f\left(v_{y, \xi}\right)} .
\end{aligned}
$$

The last equality follows from the asymptotic description of $G_{\lambda}$ in Theorem 6.10 .

We are now able obtain the following alternate description of $\nu_{x}$,

Lemma 6.12. The constant $C_{\tau}=-\left.\frac{d}{d \lambda}\right|_{\lambda=0} \tau_{\lambda}\left(v_{\mathbf{a}}\right)$ exists and is positive, and for a set $B \subset \partial M$ and the corresponding set $B_{x} \subset S_{x} M$ we have

$$
\nu_{x}(B)=\frac{1}{C_{\tau}} \int_{B_{x}} \lim _{t \rightarrow \infty} G(v, t) \mathrm{V}(v, t) d \lambda_{x}(v) .
$$

Proof. We proceed in the same way as for the proof of Lemma 3.4 but substituting the definitions of $\nu_{x}^{\lambda}$ and $n_{\lambda}(o, o)=-\frac{1}{\lambda}$ for $\mu_{x}^{s}$ and $g_{s}(o, o)$ respectively. Performing the change of variables $t \rightarrow \frac{s}{-\lambda}$ we may integrate

$$
\begin{aligned}
& \nu_{x}(B)= \lim _{\lambda \rightarrow 0^{-}}-\lambda \int_{0}^{\infty} \int_{B_{x}} G_{\lambda}(v, t) \mathrm{V}(v, t) d \lambda_{x}(v) d t \\
&=\lim _{\lambda \rightarrow 0^{-}} \int_{0}^{\infty} e^{\left(\mathrm{h}-\tau_{\lambda}\left(v_{\mathbf{a}}\right)\right) \frac{s}{-\lambda}} \times \\
& \quad \times \int_{B_{x}} G_{\lambda}\left(v, \frac{s}{-\lambda}\right) \mathrm{V}\left(v, \frac{s}{-\lambda}\right) e^{\left(\tau_{\lambda}\left(v_{\mathbf{a}}\right)-\mathrm{h}\right) \frac{s}{-\lambda}} d \lambda_{x}(v) d s .
\end{aligned}
$$

Now by Corollary $6.5, \lim _{\lambda \rightarrow 0^{-}} \frac{\mathrm{h}-\tau_{\lambda}\left(v_{\mathbf{a}}\right)}{\lambda}>\frac{1}{(n-1)(2 b-a)}$. Also by Proposition 6.4 for $s>0$,

$$
\lim _{\lambda \rightarrow 0^{-}} G_{\lambda}\left(v, \frac{s}{-\lambda}\right) \mathrm{V}\left(v, \frac{s}{-\lambda}\right) e^{\left(\tau_{\lambda}\left(v_{\mathbf{a}}\right)-\mathrm{h}\right) \frac{s}{-\lambda}}
$$

converges uniformly on compact subsets of $S_{x} M \backslash \mathcal{W}^{s}\left(v_{\mathbf{a}}\right)$. So continuing the computation,

$$
\nu_{x}(B)=\lim _{\lambda \rightarrow 0^{-}} \int_{0}^{\infty} e^{\left(\mathrm{h}-\tau_{\lambda}\left(v_{\mathbf{a}}\right)\right) \frac{s}{-\lambda}} \int_{B_{x}} \lim _{t \rightarrow \infty} G(v, t) \mathrm{V}(v, t) d \lambda_{x}(v) d s .
$$


From its definition in terms of the heat kernel,

$$
G_{\lambda}(x, y)=\int_{0}^{\infty} e^{\lambda t} p(t, x, y) d t
$$

is real analytic in $\lambda$ for $\lambda<\lambda_{1}$. It follows that $K_{\lambda}(v, t)$ is also real analytic in $\lambda$ since it by construction meromorphic but has no poles. Taking derivatives in $t$ at $t=0$ we obtain the power series expansion $\tau_{\lambda}\left(v_{\mathbf{a}}\right)=a_{0}\left(v_{\mathbf{a}}\right)+C_{\tau} \lambda+$ $a_{2}\left(v_{\mathbf{a}}\right) \lambda^{2}+\cdots$. So $\lim _{\lambda \rightarrow 0} \frac{\left(\tau_{\lambda}\left(v_{\mathbf{a}}\right)-\mathrm{h}\right)}{\lambda}=-C_{\tau}$ exists. In fact writing out the definition of $K_{\lambda}$ in terms of the heat kernel and taking derivatives we obtain,

$$
\begin{aligned}
C_{\tau}=\lim _{R \rightarrow \infty} \frac{1}{G\left(v_{\mathbf{a}}, R\right)}\left(\tau\left(v_{\mathbf{a}}\right) \int_{0}^{\infty} t p\left(t, v_{\mathbf{a}}(0), v_{\mathbf{a}}(R)\right) d t\right. \\
\left.\quad-\left.\frac{\partial}{\partial s}\right|_{s=0} \int_{0}^{\infty} t p\left(t, v_{\mathbf{a}}(s), v_{\mathbf{a}}(R)\right) d t\right) .
\end{aligned}
$$

By Lemma 6.5

$$
C_{\tau}>-\left.\frac{d}{d \lambda}\right|_{\lambda=0}\left(\sqrt{\left(\frac{n-1}{2}(2 b-a)\right)^{2}-\lambda}\right)=\frac{1}{(n-1)(2 b-a)}>0 .
$$

So we obtain,

$$
\nu_{x}(B)=\frac{1}{C_{\tau}} \int_{B_{x}} \lim _{t \rightarrow \infty} G(v, t) \mathrm{V}(v, t) d \lambda_{x}(v) d s .
$$

Directly from the formula in the above lemma we arrive at the following Lemma 6.13. Explicitly, for all $v \in S_{x} M \backslash \mathcal{W}^{s}\left(v_{\mathbf{a}}\right)$ and $x \in M$,

$$
\frac{d \nu_{x}}{d \lambda_{x}}(v)=\frac{1}{C_{\tau}} \lim _{t \rightarrow \infty} \mathrm{V}(v, t) G(v, t),
$$

and $\nu_{x}$ and $\lambda_{x}$ are absolutely continuous with respect to each other.

Corollary 6.14. $M$ is asymptotically harmonic iff $\tau=\operatorname{tr} U$ in which case the constants $C_{v_{\mathbf{a}}}, C_{\tau}$ and $C_{G}$ are related by $C_{\tau} C_{v_{\mathbf{a}}}=C_{G}$.

Proof. If $M$ is asymptotically harmonic then we have $\nu_{x}=\lambda_{x}$ in which case by Lemma 6.13 and Theorem 6.10 and Corollary 5.7, $\int_{0}^{\infty} \operatorname{tr} U\left(g^{s} v\right)-\tau\left(g^{s} v\right) d s$ is constant and since $\tau\left(v_{\mathbf{a}}\right)=\operatorname{tr} U\left(v_{\mathbf{a}}\right)$, it must be zero. It follows that $\tau(v)=\operatorname{tr} U(v)$. 
Conversely, if $\tau(v)=\operatorname{tr} U(v)$ then by the same lemmas and corollary $\frac{d \nu_{x}}{d \lambda_{x}}(v)=\frac{C_{\tau} C_{v_{\mathrm{a}}}}{C_{G}}$. Since they are both probability measures this constant is 1. Then the lemma follows from Proposition 5.15.

Using the same proof as 4.8 we obtain,

Corollary 6.15. The measure $\nu_{x}$ is absolutely continuous with its fip. Explicitly, for $v \in S_{x} M \backslash \mathcal{W}^{s}\left(v_{\mathbf{a}}\right)$,

$$
\frac{d \nu_{x}}{d F_{*} \nu_{x}}(v)=\lim _{t \rightarrow \infty} \frac{G(v, t) \mathrm{V}(v, t)}{G(-v, t) \mathrm{V}(-v, t)}
$$

From Lemmas 4.7 and 6.13 we immediately obtain

Corollary 6.16. The measures $\mu_{x}$ and $\nu_{x}$ are in the same measure class and for all $v \in S_{x} M \backslash \mathcal{W}^{s}\left(v_{\mathbf{a}}\right)$ and $x \in M$,

$$
\frac{d \nu_{x}}{d \mu_{x}}(v)=\frac{C_{M a r}}{C_{\tau}} \lim _{t \rightarrow \infty} e^{\mathrm{h} t} G(v, t) .
$$

Following the proof of Corollary 6.14 with the above corollary gives,

Corollary 6.17. $M$ is asymptotically harmonic iff $\tau(v)=\mathrm{h}$, in which case the constants $C_{M a r}, C_{\tau}$ and $C_{G}$ are related by $C_{M a r}=C_{\tau} C_{G}$.

Proposition 6.18. $M$ is asymptotically harmonic if and only if for some $x \in M, \nu_{x}, \tau$, and $\operatorname{tr} U$ are flip invariant.

Proof. If $M$ is asymptotically harmonic, then by Propositions 5.15, $\nu_{x}$ is flip invariant.

For the converse, we find from Proposition 6.15 and Theorem 6.10 that for $w \in S_{x} M \backslash\left\{ \pm d x\left(v_{\mathbf{a}}\right)\right\}$,

$$
\begin{aligned}
\frac{d \nu_{x}}{d F_{*} \nu_{x}}(w) & =\lim _{t \rightarrow \infty} \frac{\mathrm{V}(w, t) G(w, t)}{\mathrm{V}(-w, t) G(-w, t)} \\
& =e^{\int_{0}^{\infty} \operatorname{tr} U\left(-g^{s} w\right)-\operatorname{tr} U\left(-g^{s}(-w)\right)+\tau\left(g^{-s} w\right)-\tau\left(g^{-s}(-w)\right) d s} .
\end{aligned}
$$

So if $\nu_{x}$ is flip invariant, we have

$$
\int_{0}^{\infty} \operatorname{tr} U\left(-g^{s} w\right)-\operatorname{tr} U\left(g^{-s} w\right) d s=\int_{0}^{\infty} \tau\left(-g^{s} w\right)-\tau\left(g^{-s} w\right) d s
$$


By Proposition 6.4 and Corollary 2.3, these integrals make sense. Replacing $w$ by $g^{t} w$ and taking derivatives with respect to $t$ yields

$$
\operatorname{tr} U\left(g^{t} w\right)+\operatorname{tr} U\left(-g^{t} w\right)=\tau\left(g^{t} w\right)+\tau\left(-g^{t} w\right) .
$$

Combining equation 6.1 with $\operatorname{tr} U(v)=\operatorname{tr} U(-v)$ and $\tau(v)=\tau(-v)$ implies that $\tau=\operatorname{tr} U$. Hence, by Corollary $6.14, M$ is asymptotically harmonic.

Corollary 6.19. If $M$ is asymptotically harmonic then $\omega^{s}, \omega^{s s}, \omega^{u}, \omega^{s u}, \lambda, \nu$, and $\mu$ are all constant multiples.

Proof. If $M$ is asymptotically harmonic then $\tau(v)=\operatorname{tr} U(v)=\mathrm{h}$, which by Theorem 6.10 and Corollary 5.7 implies that $\lim _{t \rightarrow \infty} \mathrm{V}(v(t), v(-t)) e^{-2 \mathrm{~h} t}$ and $\lim _{t \rightarrow \infty} G(v(t), v(-t)) e^{2 \mathrm{~h} t}$ are constant. Also, $\mu_{x}=\nu_{x}=\lambda_{x}$ so from the Kaimanovich construction we have that $\lambda, \mu$, and $\nu$ are multiples. By flip invariance the other measures are all equal to $\lambda$.

Now we examine the measures $\mu, \nu, \lambda$.

Proposition 6.20. We have the following three formulas

$$
\begin{aligned}
\frac{d \mu}{d \lambda}(v) & =\lim _{t \rightarrow \infty} \frac{1}{C_{M a r}^{2}} \mathrm{~V}(v(t), v(-t)) e^{-2 \mathrm{~h} t}=\frac{C_{v_{\mathbf{a}}}}{C_{M a r}^{2}} e^{\int_{-\infty}^{\infty} \operatorname{tr} U\left(g^{t} v\right)-\mathrm{h} d t}, \\
\frac{d \nu}{d \lambda}(v) & =\frac{1}{C_{\tau}^{2}} \lim _{t \rightarrow \infty} \mathrm{V}(v(t), v(-t)) G(v(t), v(-t)) \\
& =\frac{C_{v_{\mathbf{a}}}}{C_{\tau}^{2} C_{G}} e^{\int_{-\infty}^{\infty} \operatorname{tr} U\left(g^{t} v\right)-\tau\left(g^{t} v\right) d t}
\end{aligned}
$$

and

$$
\frac{d \nu}{d \mu}(v)=\frac{C_{M a r}^{2}}{C_{\tau}^{2}} \lim _{t \rightarrow \infty} G(v(t), v(-t)) e^{2 \mathrm{~h} t}=\frac{C_{M a r}^{2}}{C_{\tau}^{2} C_{G}} e^{\int_{-\infty}^{\infty} \mathrm{h}-\tau\left(g^{t} v\right) d t},
$$

where

$$
\begin{aligned}
C_{v_{\mathbf{a}}} & =\operatorname{det}\left(U\left(v_{\mathbf{a}}\right)+U\left(-v_{\mathbf{a}}\right)\right)^{-1}, C_{\tau} \\
& =-\left.\frac{d}{d \lambda}\right|_{\lambda=0} \tau_{\lambda}\left(v_{\mathbf{a}}\right), C_{G}=\lim _{t \rightarrow \infty} G\left(v_{\mathbf{a}}, t\right) e^{t \tau\left(v_{\mathbf{a}}\right)},
\end{aligned}
$$

and $C_{M a r}$ is the Margulis constant. 
Proof. We use the definitions of the measures given in Section 3 to directly compute the stated quantities. The first equality in the first formula follows as an application of Lemma 4.7 and then by applying Corollary 5.7 for the second equality. Similarly, the first equality of the second formula results from Lemma 6.13 followed by Corollaries 5.7 and Theorem 6.10 for the second equality. To verify the last formula use Corollary 6.16 and then Theorem 6.10.

Now we may complete the proofs of the main theorems.

Theorem 1. For any NCHS M, we have the following

1. For all $x \in M$ the measures $\mu_{x}, \nu_{x}, \lambda_{x}$ and their flips are all in the same measure class.

2. The Bowen-Margulis measure $\mu$, harmonic measure $\nu$, Liouville measure $\lambda, \omega^{s s}, \omega^{s}, \omega^{s u}$, and $\omega^{u}$ are all in the same measure class, and

3. $\mathrm{h}^{2}=\operatorname{tr} \operatorname{ad}_{v_{\mathbf{a}}}^{2}=c_{M}^{2}=\beta=4 \lambda_{1}$.

Proof. By Lemmas 4.7,6.13 and Corollaries 4.8 6.15, 6.16 we have that $\nu_{x}, \mu_{x}$, and $\lambda_{x}$ and their flips are in the same measure class. First this implies that $\mu, \lambda$ and $\nu$ are in the same measure class by the Kaimanovich descriptions of these measures in terms of $\nu_{x}$ and $\mu_{x}$. Also, using the description $d \lambda(v)=$ $d \lambda_{x}(v) d m(x)$ we see that $\omega^{s s}, \omega^{s}$, and $\lambda$ are in the same measure class. By the flip quasi-invariance of the measures $\mu_{x}$ and $\nu_{x}, \omega^{s u}$ and $\omega^{s s}$ and $\omega^{u}$ and $\omega^{s}$ are in the same measure class. By transitivity the other equivalences of measures follow.

The last statement follows from Corollary 2.5, Corollary 6.6, and Proposition 2.14 .

Theorem 2. For any NCHS M, the following are equivalent

1. $\operatorname{tr} U$ and $\operatorname{tr} U^{2}$ are both flip invariant,

2. $\operatorname{tr} U$ is flip invariant and any one of $\mu_{x}, \omega^{s s}$, or $\omega^{\text {su }}$ is flip invariant,

3. $\tau$ is flip invariant and any one of $\nu_{x}, \omega^{s}$, or $\omega^{u}$ is flip invariant,

4. Any two of the measures $\mu_{x}, \nu_{x}$, or $\lambda_{x}$ coincide,

5. Any two of the measures $\lambda$, $\omega^{s s}$, or $\omega^{s}$ coincide, 
6. Any two of the measures $\lambda, \omega^{s u}$, or $\omega^{u}$ coincide,

7. Any two of $\tau(v), \mathrm{h}$, or $\operatorname{tr} U(v)$ coincide, and

8. $M$ is asymptotically harmonic.

Proof. Propositions 5.9 shows that (1) is equivalent to (8). The equivalence of (2) and (3) with (8) follows from Corollary 6.19, Propositions 4.9 and 6.18, and the definitions of $\omega^{s s}, \omega^{s}, \omega^{s u}$, and $\omega^{u}$ in Section 3. Combining Corollary 5.8 and Lemma 4.6 with Propositions 5.10 and 5.15 we obtain that (4) is equivalent to (8).

Using the definitions of $\omega^{s s}, \omega^{s u}, \omega^{s}, \omega^{u}$ and $d \lambda=d \lambda_{x} d m(x)$ item (5) is reduced to item (4). After noting that $\lambda_{x}$ is flip invariant item (6) becomes evidently equivalent to item (5). Lastly, item (7) is equivalent to (8) via Corollaries 6.14, and 6.17.

Now we will show the potentially weaker situation when the $\mu, \nu, \lambda$ agree.

Theorem 3. For any NCHS M, we have the following relationships

1. The following are all equivalent,

(a) for any $x \in M$ any one of $\frac{d \mu_{x}}{d F_{*} \mu_{x}}, \frac{d \mu_{x}}{d \lambda_{x}}$, $\frac{d \omega^{s s}}{d \lambda}$, or $\frac{d \omega^{s u}}{d \lambda}$, extends to a continuous function,

(b) for all $v \in S M, \int_{-\infty}^{\infty} \operatorname{tr} U\left(g^{t} v\right)-\mathrm{h} d t=0$,

(c) $\lambda$ is a multiple of $\mu$.

2. The following are all equivalent,

(a) for any $x \in M$ any one of $\frac{d \nu_{x}}{d F_{*} \nu_{x}}, \frac{d \nu_{x}}{d \lambda_{x}}, \frac{d \omega^{s}}{d \lambda}$, or $\frac{d \omega^{u}}{d \lambda}$, extends to a continuous function,

(b) for all $v \in S M, \int_{-\infty}^{\infty} \operatorname{tr} U\left(g^{t} v\right)-\tau\left(g^{t} v\right) d t=0$,

(c) $\lambda$ is a multiple of $\nu$.

3. The following are all equivalent,

(a) for any $x \in M$ any one of $\frac{d \nu_{x}}{d \mu_{x}}, \frac{d \omega^{s}}{d \omega^{s s}}$, or $\frac{d \omega^{u}}{d \omega^{s u}}$, extends to a continuous function,

(b) for all $v \in S M, \int_{-\infty}^{\infty} \tau\left(g^{t} v\right)-\mathrm{h} d t=0$, 
(c) $\mu$ is a multiple of $\nu$.

Proof. In case (1) by considering the expressions for the Radon-Nikodym derivatives in part (a) we find they are continuous if and only if

$$
\lim _{t \rightarrow \infty} \mathrm{V}(v, t) e^{-\mathrm{h} t}
$$

extends to a continuous function on $S M$. The expression

$$
\lim _{t \rightarrow \infty} \mathrm{V}(v(t), v(-t)) e^{-2 \mathrm{~h} t}
$$

is continuous at $v=v_{\mathbf{a}}$ if and only if $\lim _{t \rightarrow \infty} \mathrm{V}(v, t) e^{-\mathrm{h} t}$ is continuous at $v=v_{\mathbf{a}}$ since the two expressions always converge to the same value for any sequence $v_{i} \rightarrow v_{\mathbf{a}}$, hence (b) is equivalent to (a). From Proposition 6.20 we see that $\frac{d \mu}{d \lambda}(v)$ is then continuous. By G-invariance, $\frac{d \mu}{d \lambda}(v)$ must be constant (see Remark 4.11). Lastly, by Proposition 6.20,

$$
\int_{-\infty}^{\infty} \operatorname{tr} U\left(g^{t} v\right)-\mathrm{h} d t=0
$$

if and only if $\lambda$ is a multiple of $\nu$ (Recall that $\operatorname{tr} U\left(g^{t} v_{\mathbf{a}}\right)=\tau\left(g^{t} v_{\mathbf{a}}\right)=\mathrm{h}$ so the integral is always 0 in the abelian direction).

The second and third cases follow from Proposition 6.20 similarly except that we use respectively, $\lim _{t \rightarrow \infty} \mathrm{V}(v, t) G(v, t)$ and $\lim _{t \rightarrow \infty} G(v, t) e^{\mathrm{h} t}$ in place of $\lim _{t \rightarrow \infty} \mathrm{V}(v, t) e^{-\mathrm{h} t}$. The continuity of these imply that $\lim _{t \rightarrow \infty} G(v(t), v(-t)) \mathrm{V}(v(t), v(-t))$ and $\lim _{t \rightarrow \infty} G(v(t), v(-t)) e^{2 \mathrm{~h} t}$ are respectively continuous at $v_{\mathbf{a}}$ (and everywhere else by G-equivariance). Again using the Kaimanovich construction we obtain $\nu=\lambda$ and $\mu=\nu$ respectively.

\section{Examples of Negatively Curved Homogeneous Spaces which are not Asymptotically Harmonic.}

Here we present a class of examples of NCHS's which are not asymptotically harmonic. This emphasizes the differences between NCHS's and the compact case, since Theorem 2 states that none of the listed geometric measures may coincide, but by Theorem 1 they are all in the same measure class.

We refer the reader at this point to Section 4 of [18] for the details of the construction of amalgamated products of homogeneous spaces.

Let $M_{1}$ and $M_{2}$ be general NCHS's. Then we can form the amalgamated product $M=M_{1} \# M_{2}$ of $M_{1}$ and $M_{2}$. For simplicity, we will identify $M$, 
$M_{1}=A \ltimes N_{1}$ and $M_{2}=A \ltimes N_{2}$ with their corresponding transitive group of isometries. The Lie algebra of $M$ is $\mathfrak{g}=\mathbb{R} v_{\mathbf{a}} \oplus\left(\mathfrak{n}_{1} \oplus \mathfrak{n}_{2}\right)$ where the action of $\operatorname{ad}_{v_{\mathfrak{a}}}$ on $\mathfrak{n}_{i}$ is the same as in the respective $M_{i}$ and $\left[\mathfrak{n}_{1}, \mathfrak{n}_{2}\right]=0$. The left invariant metric on $M$ is uniquely determined from the metrics on the $\mathfrak{n}_{i}$ and the conditions that $\left|v_{\mathbf{a}}\right|=1$ and the direct sum in $\mathfrak{g}$ be orthogonal. For our examples below we will need the fact that $M_{i}$ are both isometrically embedded totally geodesic submanifolds in $M_{1} \# M_{2}$. This is Proposition 4.3 of [18].

For our examples we restrict to the case where $M_{1}$ is any asymptotically harmonic NCHS of dimension $n$ and $M_{2}=\mathbb{H}_{b}^{2}$, the real hyperbolic plane of constant curvature $-b^{2}$ for any $b>0$.

Now choose any unit vector $v \in S_{e} M_{1} \subset T_{e} M$. Then let $v_{o}(t)=$ $d(v(t))^{-1} g^{t} v$ be the pullback of the geodesic tangent vector $v^{\prime}(t)$ by isometries to $S_{o} M \subset \mathfrak{g}$. Since $M_{1}$ is totally geodesic in $M, v_{o}(t)$ stays in $S_{o} M_{1}$. By the Lie algebra structure of $\mathfrak{g}, \operatorname{ad}_{v_{\mathbf{a}}} Y=b \cdot Y$ for $Y \in T_{e} N_{2}$. Formula 2.1 then shows that $\nabla_{v_{\mathbf{a}}} Y=0$ and $\nabla_{X} Y=\nabla_{Y} X=0$ for $X \in T_{e} N_{1}$ and $Y \in T_{e} N_{2}$. Consequently, the left invariant vector field $Y$ is parallel along $v(t)\left(\nabla_{v(t)} Y=\right.$ $0)$. Denote the curvature tensor of $M$ and $M_{1}$ by $R$ and $R_{1}$ respectively. Since $M_{1}$ sits in $M$ as a totally geodesic submanifold, the curvature tensor satisfies $R\left(v_{o}(t), X, v_{o}(t), Y\right)=R_{1}\left(v_{o}(t), X, v_{o}(t), Y\right)$ for all $X, Y \in T_{o} M_{1}$. Also, we may compute directly from the definition of curvature using the connection formula 2.1 (or using the formula with opposite sign in Section 4.5 of [18]) that $R\left(v_{o}(t), X, v_{o}(t), Y\right)=R\left(v_{o}(t), v_{\mathbf{a}}, v_{o}(t), Y\right)=0$ and $R\left(v_{o}(t), Y, v_{o}(t), Y\right)=-b^{2}\left\langle v_{o}(t), v_{\mathbf{a}}\right\rangle^{2}-b\left\langle\operatorname{ad}_{v_{\mathbf{a}}} v_{o}(t), v_{o}(t)\right\rangle$, for all $X \in T_{e} N_{1}$ and $Y \in T_{e} N_{2}$. Now let $\left\{P_{i}(t)\right\}_{i=1}^{n}$ be a parallel orthonormal base in $M$ for $v(t)^{\perp}$ pulled back via isometries to the origin $o$ such that $P_{n}(t)=P_{n}(0) \in$ $T_{e} N_{2}$ and $P_{i}(t) \in T_{e} M_{1}$ for $i=1, \ldots, n-1$ and all $t \in \mathbb{R}$. The above calculations along with the symmetry of the curvature tensor allow us to compute the curvature tensor in the base $\left\{P_{i}\right\}$ to be of block form

$$
\begin{aligned}
R\left(g^{t} v\right) & =R\left(v_{o}(t), \cdot, v_{o}(t), \cdot\right) \\
& =\left(\begin{array}{cc}
R_{1}\left(v_{o}(t), \cdot, v_{o}(t), \cdot\right) & 0 \\
0 & -b^{2}\left\langle v_{o}(t), v_{\mathbf{a}}\right\rangle^{2}-b\left\langle\operatorname{ad}_{v_{\mathbf{a}}} v_{o}(t), v_{o}(t)\right\rangle
\end{array}\right) .
\end{aligned}
$$

Since $R(t)$ is zero off the diagonal blocks, it follows from the Riccati equation $\dot{U}\left(g^{t} v\right)=R\left(g^{t} v\right)+U\left(g^{t} v\right)^{2}$ that $U\left(g^{t} v\right)$ has the block form

$$
U\left(g^{t} v\right)=\left(\begin{array}{cc}
U_{1}\left(g^{t} v\right) & 0 \\
0 & u_{2}(t)
\end{array}\right)
$$

where $U_{1}\left(g^{t} v\right)$ is the second fundamental form for horospheres in $M_{1}$ and 
$u_{2}(t)$ is the solution to the scalar Riccati equation $\dot{u}_{2}(t)=-b^{2}\left\langle v_{o}(t), v_{\mathbf{a}}\right\rangle^{2}-$ $b\left\langle\operatorname{ad}_{v_{\mathbf{a}}} v_{o}(t), v_{o}(t)\right\rangle+u_{2}(t)^{2}$.

Since we assumed $M_{1}$ to be asymptotically harmonic, $\operatorname{tr} U_{1}\left(g^{t} v\right)=$ $\mathrm{h}_{1}$ for some constant $\mathrm{h}_{1}>0$. Hence $\operatorname{tr} U\left(g^{t} v\right)=\operatorname{tr} U_{1}\left(g^{t} v\right)+u_{2}(t)$ is constant if and only if $u_{2}(t)$ is constant. Now if $v \neq \pm v_{\mathbf{a}}$ then $\lim _{t \rightarrow \infty} d\left(g^{t} v, g^{t} w_{v}^{+}\right)=0$ in the Sasaki metric by the definition of $w_{v}^{+}$(see Section 2). This shows that either $v_{o}(t)= \pm v_{\mathrm{a}}$ or else as $v_{o}(t)$ converges to $\pm v_{\mathbf{a}}$ as $t \rightarrow \infty$ causing $-b^{2}\left\langle v_{o}(t), v_{\mathbf{a}}\right\rangle^{2}-b\left\langle\operatorname{ad}_{v_{\mathbf{a}}} v_{o}(t), v_{o}(t)\right\rangle$ to approach $-b^{2}$ in either case. Therefore $u_{2}(t)$ is constant if and only if $u_{2}(t)=b$ and $b^{2}\left\langle v_{o}(t), v_{\mathbf{a}}\right\rangle^{2}+b\left\langle\operatorname{ad}_{v_{\mathbf{a}}} v_{o}(t), v_{o}(t)\right\rangle=b^{2}$. This in turn implies that if $v_{o}(t)=\alpha(t) v_{\mathbf{a}}+\beta(t) X(t)$ with $X(t) \in S_{o} N_{1}$ and $\alpha(t)^{2}+\beta(t)^{2}=1$ then $\left\langle\operatorname{ad}_{v_{\mathrm{a}}} X(t), X(t)\right\rangle=b$. Since the choice of initial vector $v \in S_{o} M_{1}$ was arbitrary, $\left.\left(\operatorname{ad}_{v_{\mathbf{a}}}+\operatorname{ad}_{v_{\mathbf{a}}}^{*}\right)\right|_{\mathfrak{n}_{1}}=b \cdot$ Id. This implies by Proposition 2.1 of [18] that $M_{1}$ has constant curvature $-b^{2}$ and hence $M$ is a hyperbolic space of dimension $n+1$. Simply stated, whenever $M_{1}$ does not have constant curvature $-b^{2}, M$ is not asymptotically harmonic.

\section{References.}

[1] A. V. Alekseevskiî, Homogeneous Riemannian spaces of negative curvature, Mat. Sb. (N.S.) 96(138) (1975), 93-117.

[2] A. Ancona, Negatively curved manifolds, elliptic operators, and the Martin boundary, Ann. of Math. 125 (1987), 495-536.

[3] Hadamard, Comment. Math. Helv. 64 (1989), 62-83.

[4] M. T. Anderson and R. Schoen, Positive harmonic functions on complete manifolds of negative curvature, Ann. of Math. 121 (1985), 429-446.

[5] R. Azencott and E. Wilson, Homogeneous manifolds with negative curvature, $i$, Trans. Amer. Math. Soc. 215 (1976), 323-362.

[6] Homogeneous manifolds with negative curvature, ii, Mem. Amer. Math. Soc. 178 (1976).

[7] M. Babillot, Comportement asymptotique du mouvement brownien sur une variété homogène à courbure négative ou nulle, Ann. Inst. H. Poincaré Probab. Statist. 27 (1991), no. 1, 61-90.

[8] W. Ballmann, Lectures on spaces of nonpositive curvature, DMV Seminar, vol. 25, Birkhäuser Verlag, Basel, 1995, With an appendix by Misha Brin. 
[9] W. Ballmann, M. Brin, and P. Eberlein, Structure of manifolds of nonpositive curvature, I, Ann. of Math. 122 (1985), 171-203.

[10] Y. Benoist, F. Foulon, and F. Labourie, Flots d'Anosov à distributions stable et instable différentiables, J. Amer. Math. Soc. 5 (1992), 33-74.

[11] A. L. Besse, Manifolds all of whose geodesics are closed, Ergebnisse der Mathematik und ihrer Grenzgebiete [Results in Mathematics and Related Areas], vol. 93, Springer-Verlag, Berlin, 1978, With appendices by D. B. A. Epstein, J.-P. Bourguignon, L. Bérard-Bergery, M. Berger and J. L. Kazdan.

[12] G. Besson, G. Courtois, and S. Gallot, Entropies et rigidités des espaces localement symétriques de courbure strictement négative, GAFA 5 (1995), 731-799.

[13] R. Brooks, A relation between growth and the spectrum of the Laplacian, Math. Z. 178 (1981), no. 4, 501-508.

[14] I. Chavel, Riemannian geometry-a modern introduction, Cambridge Tracts in Mathematics, vol. 108, Cambridge University Press, Cambridge, 1993.

[15] I. Chavel, B. Randol, and J. Dodziuk, Eigenvalues in Riemannian geometry, Academic Press, Inc., 1984.

[16] J. Cheeger, A lower bound for the smallest eigenvalue of the Laplacian, Problems in analysis (Papers dedicated to Salomon Bochner, 1969) (Princeton, N. J.), Princeton Univ. Press, 1970, 195-199.

[17] E. Damek and F.Ricci, A class of non-symmetric harmonic Riemannian spaces, Bull. Amer. Math. Soc. (N.S.) 27 (1992), 139-142.

[18] P. Eberlein and J. Heber, Quarter pinched homogeneous spaces of negative curvature, Internat. J. Math. 7 (1996), no. 4, 441-500.

[19] H. Federer, Geometric measure theory, Springer-Verlag New York Inc., New York, 1969.

[20] R. Feres, Metric entropy rigidity after Besson, Courtois, and Gallot, Notes, 1994.

[21] G. B. Folland, Real analysis. modern techniques and their applications, Pure and Applied Mathematics, John Wiley \& Sons Inc., New York, 1984, A WileyInterscience Publication.

[22] P. Foulon and F. Labourie, Sur les variétés compactes asymptotiquement harmoniques, Invent. Math. 109 (1992), 97-111.

[23] L. Garnett, Foliations, the ergodic theorem and Brownian motion, J. Func. Anal. 51 (1983), 285-311. 
[24] U. Hamenstädt, A new description of the Bowen-Margulis measure, Ergodic Theory Dynamical Systems 9 (1989), 455-464.

[25] An explicit description of harmonic measure, Math. Z. 205 (1990), 287-299.

[26] Ursula Hamenstädt, Regularity at infinity of compact negatively curved manifolds, Ergodic Theory Dynam. Systems 14 (1994), no. 3, 493-514.

[27] J. Heber, Homogeneous spaces of nonpositive curvature and their geodesic flow, Internat. J. Math. 6 (1994), no. 2, 279-296.

[28] E. Heintze, Compact quotients of homogeneous negatively curved Riemannian manifolds, Math. Z. 140 (1974), 79-80.

[29] , On homogeneous manifolds of negative curvature, I, Math. Ann. 211 (1974), 23-34.

[30] E. Heintze and H. C. Im Hof, Geometry of horospheres, J. Differential Geom. 12 (1977), 481-491.

[31] K. Jacobs, Measure and integral, Academic Press [Harcourt Brace Jovanovich Publishers], New York, 1978, Probability and Mathematical Statistics, With an appendix by Jaroslav Kurzweil.

[32] V. A. Kaumanovich, Note on brownian motion paper, Personal Communication.

[33] _ An entropy criterion of maximality for the boundary of random walks on discrete groups, Soviet Math. Doklady 31 (1985), no. 5, 1051-1054.

[34] V. A. Kaimanovich, Brownian motion and harmonic functions on covering manifolds. an entropy approach, Soviet Math. Doklady 33 (1986), 812-816.

[35] V. A. Kalmanovich, Invariant measures of the geodesic flow and measures at infinity on negatively curved manifolds, Ann. Inst. Henri Poincaré Physique Théorique 53 (1990), no. 4, 361-393.

[36] F. Ledrappier, Propriété de poisson et courbure négative, C.R.A.S. Paris 305 (1987), 191-194.

[37] Ergodic properties of Brownian motion on covers of compact negatively curved manifolds, Bol. Soc. Bras. Mat. 19 (1988), 115-140. Harmonic measures and Bowen-Margulis measures, Israel Journal of Mathematics 71 (1990), no. 3, 275-287.

[39] F. Ledrappier, A heat kernel characterization of asymptotic harmonicity, Proc. Amer. Math. Soc. 118 (1993), no. 3, 1001-1004. 
[40] P. Mattila, Geometry of sets and measures in Euclidean spaces. fractals and rectifiability, Cambridge Studies in Advanced Mathematics, vol. 44, Cambridge University Press, Cambridge, 1995.

[41] J.-J. Prat, Étude asymptotique et convergence angulaire du mouvement brownien sur une variété à courbure négative, C. R. Acad. Sci. Paris Sér. A-B 280 (1975), no. 22, Aiii, A1539-A1542.

[42] D. Sullivan, The density at infinity of a discrete group of hyperbolic motions, Inst. Hautes Études Sci. Publ. Math. 50 (1979), 225-250.

[43] The Dirichlet problem at infinity for a negatively curved manifold, J. Differential Geom. 18 (1983), 723-732.

[44] Z. Szabó, The Lichnerowicz conjecture on harmonic manifolds, J. Differential Geom. 31 (1990), 1-28.

[45] J. Wolf, Homogeneity and bounded isometries in manifolds of negative curvature, Illinois J. Math 8 (1964), 14-18.

[46] T. Wolter, Einstein metrics on solvable groups, Math. Z. 206 (1991), 457-471. Math. 2 (1991), 223-234.

[48] C. B. Yue, Integral formulas for the Laplacian along the unstable foliation, Ergodic Theory Dynamical Systems 11 (1991), 803-819.

[49] _ On Sullivan's conjecture, Random and Computational Dynamics 1 (1992), 131-145.

[50] , Rigidity and dynamics around manifolds of negative curvature, Math. Res. Lett. 1 (1994), 123-147.

UNIVERSity of Illinois, ChicAgo

Chicago, IL 60607

E-mail address: cconnell@math.uic.edu

Received May 11, 1998. 